\title{
Electricity spot price modeling by multi-factor uncertain process: a case study from the Nordic region
}

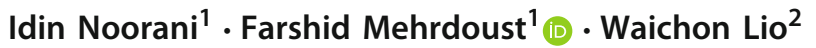 \\ Accepted: 23 July 2021 / Published online: 6 August 2021 \\ (c) The Author(s), under exclusive licence to Springer-Verlag GmbH Germany, part of Springer Nature 2021
}

\begin{abstract}
In recent years, the liberalization of energy markets (especially electricity) by many countries has led to much attention being paid to their modeling. The energy market modeling under the framework of probability theory is valuable when the distribution function is close enough to the actual frequency. However, due to the complexity and variability of the world, economic reasons and changing government policies, this assumption is not applicable in some cases. Under such circumstances, we propose an uncertain two-factor model based on uncertain differential equations to evaluate the electricity spot price dynamics. Then, several essential indicators of electricity are investigated and generalized moment estimation for unknown parameters is also provided. Two case studies by using electricity data from the Oslo and Stockholm regions illustrate our approach. We also compare the proposed model with one-factor uncertain model driven by Liu process and the electricity stochastic model. A detailed numerical study illustrates the efficiency of the proposed model to evaluate electricity spot prices.
\end{abstract}

Keywords Electricity market $\cdot$ Moment estimations $\cdot$ Multi-factor model $\cdot$ Uncertain theory

\section{Introduction}

Uncertain theory and probability theory are two axiomatic mathematical systems to rationally handle the indeterminacy. It was demonstrated by Liu (2012a) that probability theory is suitable to deal with frequency, whereas uncertainty theory is suitable to deal with belief degree. For a given quantity, a distribution function should be given in order to select whether probability theory or uncertainty theory is better to be used. Liu (2019) provided a convincing example based on uncertain urn problem to illustrate that probability theory should be applied if the given distribution function is close enough to the frequency; otherwise, uncertainty theory has to be applied. Unfortunately, the given distribution function is not close enough to the real frequency in many practical situations. Therefore, uncertainty theory is

\section{Farshid Mehrdoust}

far.mehrdoust@gmail.com

1 Department of Applied Mathematics, Faculty of Mathematical Sciences, University of Guilan, P. O. Box: 41938-1914, Rasht, Iran

2 Department of Mathematical Sciences, Tsinghua University, Beijing 100084, China an important mathematical tool to deal with the real problems. Major topics of uncertainty theory include uncertain programming (Liu 2009a), uncertain statistics (Liu 2010a), uncertain risk analysis and reliability analysis (Liu 2010b), uncertain propositional logic (Li and Liu 2009), uncertain set (Liu 2010c), uncertain logic (Liu 2011), uncertain inference (Liu 2010c), uncertain process (Liu 2008), uncertain calculus (Liu 2009b), and uncertain differential equation (Liu 2008).

As an important application of uncertainty theory, uncertain differential equation has been developed by many scholars. An existence and uniqueness theorem of solutions of uncertain differential equations was verified by Chen and Liu (2010) under the assumption of linear growth and Lipschitz condition, while the theorem is verified by Gao (2012) under the assumption of local linear growth and Lipschitz condition. Moreover, Chen and Liu (2010) presented a method to get the analytic solutions of linear uncertain differential equations, and Liu (2012b) and Yao (2013b) obtained the analytic solutions of some specific types of nonlinear uncertain differential equations. Following that, a vital numerical method for calculating the solution of an uncertain differential equation by a family of solutions of ordinary differential equations was introduced by Yao and Chen (2013). Based on this numerical method, Yao (2013a) further com- 
puted the extreme value, first hitting time and time integral of the solutions of uncertain differential equations. Another concern about uncertain differential equation is its parameter estimation based on the some given observed values. For this purpose, the method of moments (Yao and Liu 2020), least squares estimation (Sheng et al. 2020), generalized moment estimation (Liu 2021), uncertain maximum likelihood (Liu and Liu 2020) and minimum cover estimation (Yang et al. 2020) were applied. Furthermore, the initial value estimation of uncertain differential equation was investigated by Lio and Liu (2020).

Nowadays, uncertain differential equation has been successfully used in COVID-19 spread (Chen et al. 2020; Jia and Chen 2020; Lio and Liu 2020), finance (Mehrdoust and Najafi 2020; Hassanzadeh and Mehrdoust 2018; Liu 2013), pharmacokinetics (Liu and Yang 2021), population growth (Zhang and Yang 2020), heat conduction (Yang and Yao 2017), string vibration (Gao and Ralescu 2019) and spring vibration (Jia and Yang 2018). This paper aims to develop the application of uncertain differential equation to the field about electricity market.

To maintain a balance between production and demand, all manufacturers must follow the National Network Company (NGC) regulatory guidelines. The prices paid for this purpose are determined by Pool rules. Every day, pursuant to the existing capacities, the producers offer the prices of each production set for the next day. Then, to estimate the demand forecast level based on the bid price with lowest cost, NGC uses a computer algorithm to determine the operational plan (see Green 1996). In most deregulated electricity markets, there is a day-ahead market. In the Nordic region, the day-ahead market is a non-mandatory market called Elspot, which organized by Nord Pool. The significant examples of non-mandatory day-ahead markets are APX Power UK, Powernext and European Energy Exchange. In these markets, daily hourly electricity contracts are traded for physical delivery over the next 24 hours (midnight to midnight). On the Nord Pool's spot market, Nordic countries players (Danish, Finnish, Norwegian and Swedish) trade hourly contracts for each of the next 24 hours (see Benth and Koekebakker 2008). Every morning, players make offers to buy or sell a certain amount of electricity for different hours of the next day. When the spot market closes, at noon each day, the dayahead price is obtained for each hour of the next day (for more information, we refer readers to Benth et al. 2008). Asian and European options are two type options which are traded in this market. Asian options in the Nord Pool market are always written on the spot price (see Weron 2008). However, as noted by Mehrdoust and Noorani (2021), these options were traded on the Nord Pool energy exchange in the 1990s and they abandoned in 2000. Nevertheless, European-style options are very common in the Nord Pool market and are still traded on future contracts (see Benth and Kruhner 2015). Spark-spread option is another important class of derivatives in the electricity and gas market. These derivatives are written in various electricity and gas future contracts, and factory owners can use this type of derivatives for hedging the undesirable movements that occur in the gas and electricity markets. Valuation of spark-spread option was recently studied by Carmona et al. (2013).

With the liberalization of electricity markets, most of the literature in the electricity spot price is to develop models that are more consistent with reality market. In recent years, researchers have modeled the dynamics of daily electricity prices in the Nordic power exchange using various characteristics such as seasonality, mean reversion, jumps and regime-switching processes. For instance, in order to motivate the Heath-Jarrow-Morton approach to evaluate swap prices, Weron (2008) provided a thorough discussion of how the Nordic energy market is organized. Lucia and Schwartz (2002) proposed two models to describe the stochastic process governing the spot price in the Nordic power exchange. The first model is a one-factor model with two components: a known deterministic function of time and a stochastic process that is either a mean-reverting process or a Ornstein-Uhlenbeck process. The second model is a two-factor model with three components: a known deterministic function of time, a mean-reverting process and a drifted Brownian motion. Geman and Roncoroni (2006) introduced a similar model through jump process for electrical spikes. Empirical results showed that their proposed model has good flexibility and it is consistent with the observed electricity spot prices in several markets. Mehrdoust and Noorani (2021) modeled the electricity spot price in the Nordic power exchange by using a deterministic component, which is a function of time, and a stochastic component. The stochastic component was modeled by a mean-reverting process, drifted Brownian motion and jump component, such that the model parameters were dependent on hidden Markov chain. In addition to fitting Nord Pool market prices, their model also has the ability to generate forward contract prices. Other works on spot price models include, to mention a few, BarndorffNielsen et al. (2018), Bennedsen (2017), Mayer et al (2015), Liebl (2013), Rypdal and Løvsletten (2013), Benth et al. (2012) and Janczura and Weron (2010).

An acceptable comparison between the actual market data and the obtained data by the implemented model in the framework of probability theory requires that the distribution function of the considered stochastic model is close enough to the actual frequency (see Liu 2015). However, given that unforeseen events (system failure, climate change, war, etc.) and economic problems (inflation and recession) affect the behavior of financial and energy time series, this assumption is not applicable in some cases. In such a situation, to better deal with dynamic noises in the electricity spot price, this paper uses uncertain differential equations under 
the framework of uncertainty theory. As far as we know, there are no papers in the literature electricity modeling when the electricity spot price is modeled by the uncertain process. This paper intends to fill this gap by concentrating upon the two-factor canonical Liu processes. In this work, first the model parameters are estimated by applying the generalized moment estimation method. We then show that the electricity spot prices obtained by two-factor model are closer to market realities compared to the one-factor model.

The rest of this paper is organized as follows. In Sect. 2, some concepts and theorems about uncertain variables and uncertain differential equations are introduced. In Sect. 3, we formulate and explain the electricity model in the uncertain framework. In Sect. 4, we estimate the model parameters by employing generalized moment estimation method based on the $\alpha$-path of the uncertain differential equations. Section 5 reports our numerical results, and Sect. 6 contains our conclusions.

\section{Preliminary}

In this section, the basic definitions and fundamental concepts in uncertain theory including uncertain axioms, uncertain process and uncertain differential equation are introduced.

Definition 1 (Liu 2007) Let $\mathcal{L}$ be a $\sigma$-algebra on a non-empty set $\Gamma$. A set function $\mathcal{M}: \mathcal{L} \longrightarrow[0,1]$ is called an uncertain measure if it satisfies the following axioms

- (Normality Axiom) $\mathcal{M}\{\Gamma\}=1$ for the universal set $\Gamma$.

- (Duality Axiom) $\mathcal{M}\{\Lambda\}+\mathcal{M}\left\{\Lambda^{C}\right\}=1$ for any event $\Lambda$.

- (Subadditivity Axiom) For every countable sequence of events $\Lambda_{1}, \Lambda_{2}, \ldots$, we have

$$
\mathcal{M}\left\{\bigcup_{i=1}^{\infty} \Lambda_{i}\right\} \leq \sum_{i=1}^{\infty} \mathcal{M}\left\{\Lambda_{i}\right\}
$$

The triple $(\Gamma, \mathcal{L}, \mathcal{M})$ is called an uncertainty space.

- (Product Axiom) Let $\left(\Gamma_{i}, \mathcal{L}_{i}, \mathcal{M}_{i}\right)$ be an uncertainty space for $i=1,2, \ldots, n$. Then, the product uncertain measure $\mathcal{M}$ is an uncertain measure on product $\sigma$-algebra $\mathcal{L}_{1} \times \mathcal{L}_{2} \times \ldots \times \mathcal{L}_{n}$ satisfying

$\mathcal{M}\left\{\prod_{i=1}^{n} \Lambda_{i}\right\}=\min _{1 \leq i \leq n} \mathcal{M}\left\{\Lambda_{i}\right\}$,

where $\Lambda_{i}$ are arbitrarily chosen events from $\mathcal{L}_{i}$ for $i=$ $1,2, \ldots, n$, respectively.
Definition 2 (Liu 2007) If $\xi$ is measurable from $(\Gamma, \mathcal{L}, \mathcal{M})$ to a real number set, i.e., the following set

$\{\xi \in \mathcal{B}\}=\{\gamma \in \Gamma \mid \xi(\gamma) \in \mathcal{B}\}$

is an event for each real Borel set $\mathcal{B}$, then $\xi$ is called an uncertain variable.

Definition 3 (Liu 2007) An uncertainty distribution of the uncertain variable $\xi$ is defined as

$\Phi(x)=\mathcal{M}\{\xi \leq x\}$,

for arbitrary real number $x$; then, $x$ is called an uncertain variable.

Definition 4 (Liu 2009b) An uncertain variable sequence of $\xi_{1}, \xi_{2}, \ldots, \xi_{n}$ is regarded to be independent mutually if

$\mathcal{M}\left\{\bigcap_{i=1}^{n}\left(\xi_{i} \in \mathcal{B}_{i}\right)\right\}=\bigwedge_{i=1}^{n} \mathcal{M}\left\{\xi_{i} \in \mathcal{B}_{i}\right\}$

is tenable for any real Borel sets $\mathcal{B}_{1}, \mathcal{B}_{2}, \ldots, \mathcal{B}_{n}$.

Definition 5 (Liu 2015) An uncertain variable $\xi$ is called normal if it has a normal uncertainty distribution

$\Phi(x)=\left(1+\exp \left(\frac{\pi(\mu-x)}{\sqrt{3} \sigma}\right)\right)^{-1}, \quad x \in \mathbb{R}$,

denoted by $\mathcal{N}(\mu, \sigma)$ where $\mu$ and $\sigma$ are real numbers with $\sigma>0$. Besides, an uncertain variable is called lognormal if $\ln \xi$ is a normal uncertain variable $\mathcal{N}(\mu, \sigma)$.

Theorem 1 (Liu 2015) Let $\xi$ be an uncertain variable with continuous uncertainty distribution $\Phi$. Then, for any interval $[a, b]$, we have

$\Phi(b)-\Phi(a) \leq \mathcal{M}\{a \leq \xi \leq b\}$.

Theorem 2 (Liu 2015) Let $\xi_{1}$ and $\xi_{2}$ be independent normal uncertain variables $\mathcal{N}\left(\mu_{1}, \sigma_{1}\right)$ and $\mathcal{N}\left(\mu_{2}, \sigma_{2}\right)$, respectively. Then, the sum $\xi_{1}+\xi_{2}$ is also a normal uncertain variable $\mathcal{N}\left(\mu_{1}+\mu_{2}, \sigma_{1}+\sigma_{2}\right)$, i.e.,

$\mathcal{N}\left(\mu_{1}, \sigma_{1}\right)+\mathcal{N}\left(\mu_{2}, \sigma_{2}\right)=\mathcal{N}\left(\mu_{1}+\mu_{2}, \sigma_{1}+\sigma_{2}\right)$.

Definition 6 (Liu 2007) Let $\xi$ be an uncertain variable with uncertainty distribution $\Phi$. Then, the expected value of $\xi$ is defined by

$\mathbb{E}[\xi]=\int_{0}^{+\infty} \mathcal{M}\{\xi \geq x\} \mathrm{d} x-\int_{-\infty}^{0} \mathcal{M}\{\xi \leq x\} \mathrm{d} x$, 
provided that at least one of the two integrals is finite. Besides, the variance of $\xi$ is defined by $V[\xi]=\mathbb{E}[(\xi-$ $\left.\mathbb{E}[\xi])^{2}\right]$.

Theorem 3 (Liu 2015) If $\xi$ has an inverse uncertainty distribution $\Phi^{-1}(\alpha)$, and $k$ be a positive integer, then the $k$ th moment of $\xi$ is as follows:

$\mathbb{E}\left[\xi^{k}\right]=\int_{0}^{1}\left(\Phi^{-1}(\alpha)\right)^{k} \mathrm{~d} \alpha$

In the other word, for a standard normal uncertain variable $\xi \sim \mathcal{N}(0,1)$, we have

$E\left[\xi^{k}\right]=\left(\frac{\sqrt{3}}{\pi}\right)^{k} \int_{0}^{1}\left(\ln \frac{\alpha}{1-\alpha}\right)^{k} \mathrm{~d} \alpha$.

Definition 7 (Liu 2008) Let $(\Gamma, \mathcal{L}, \mathcal{M})$ be an uncertainty space and let $T$ be a totally ordered set (e.g., time). An uncertain process is a function $C_{t}(\gamma)$ from $T \times(\Gamma, \mathcal{L}, \mathcal{M})$ to the set of real numbers such that $C_{t} \in B$ is an event for any Borel set $B$ of real numbers at each $t \in T$.

Definition 8 (Liu 2009b) An uncertain process $C_{t}$ is called a Liu process if

- $C_{0}=0$ and almost all sample paths are Lipschitz continuous,

- $C_{t}$ has stationary and independent increments,

- the increment $C_{s+t}-C_{s}$ has a normal uncertainty distribution

$$
\Phi_{t}(x)=\left(1+\exp \left(-\frac{\pi x}{\sqrt{3} t}\right)\right)^{-1}, \quad x \in \mathbb{R} .
$$

Let $C_{t}$ be an uncertain process. For any partition of closed interval $[a, b]$ with $a=t_{1}<t_{2}<\ldots<t_{k+1}=b$, the mesh is written as

$\Delta=\max _{1 \leq i \leq k}\left|t_{i+1}-t_{i}\right|$

Then, the time integral of $C_{t}$ with respect to $t$ is

$\int_{a}^{b} C_{t} \mathrm{~d} t=\lim _{\Delta \rightarrow 0} \sum_{i=1}^{k} C_{t_{i}} \cdot\left(t_{i+1}-t_{i}\right)$,

provided that the limit exists almost surely and is finite. $C_{t}$ is said to be time integrable.

Definition 9 (Li and Liu 2009) Suppose that $C_{t}$ is a Liu process, and $f$ and $g$ are two measurable real functions. Then,

$\mathrm{d} X_{t}=f\left(t, X_{t}\right) \mathrm{d} t+g\left(t, X_{t}\right) \mathrm{d} C_{t}$,

is called an uncertain differential equation.
Definition 10 (Yao and Chen 2013) Let $0<\alpha<1$ be a real number. An uncertain differential equation (1) has an $\alpha$ path $X_{t}^{\alpha}$, if it solves the corresponding ordinary differential equation as follows:

$\mathrm{d} X_{t}^{\alpha}=f\left(t, X_{t}^{\alpha}\right) \mathrm{d} t+\left|g\left(t, X_{t}^{\alpha}\right)\right| \Phi^{-1}(\alpha) \mathrm{d} t$,

where $\Phi^{-1}(\alpha)$ is the inverse standard normal uncertainty distribution, i.e.,

$\Phi^{-1}(\alpha)=\frac{\sqrt{3}}{\pi} \ln \frac{\alpha}{1-\alpha}$.

In this case, $X_{t}$ is called a contour process.

Theorem 4 (Yao and Chen 2013) Let $X_{t}$ and $X_{t}^{\alpha}$ be the solution and $\alpha$-path of the uncertain differential equation

$\mathrm{d} X_{t}=f\left(t, X_{t}\right) \mathrm{d} t+g\left(t, X_{t}\right) \mathrm{d} C_{t}$,

respectively. Then, for any monotone function $J$, we have

$\mathbb{E}\left[J\left(X_{t}\right)\right]=\int_{0}^{1} J\left(X_{t}^{\alpha}\right) \mathrm{d} \alpha$.

Theorem 5 (Yao and Chen 2013) Let $X_{t}$ and $X_{t}^{\alpha}$ be the solution and $\alpha$-path of the uncertain differential equation (1), respectively. Then,

$\mathcal{M}\left\{X_{t} \leq X_{t}^{\alpha}, \forall t\right\}=\alpha$,

$\mathcal{M}\left\{X_{t}>X_{t}^{\alpha}, \forall t\right\}=1-\alpha$.

This theorem is called Yao-Chen formula.

\section{Multi-factor model of electricity}

It is significant to allow seasonal changes, because the demand for energies, especially electricity, varies with temperatures, which are highly dependent on season. The idea of modeling is to first identify a seasonal floor as $f$, such that prices are returning to it (see Benth et al. 2008). According to this approach, the floor can be obtained by fitting the deterministic function to data, and then, move the whole function down until the difference between the observations of energy spot price and floor is positive. The difference is referred to as the "deseasonalized" spot prices. From Benth et al. (2008), since the electricity spot prices show a definite pattern between specific months, seasonal behavior can be modeled with a simple sinusoidal function as follows:

$f_{t}=a+b t+c \sin \left(\frac{2 \pi(t-d)}{365}\right)$ 
for constants $a, b, c$ and $d$. Suppose that the spot price dynamics $S(t), 0 \leq t \leq T$, with maturity date $T>0$ is defined by

$S_{t}=g\left(t, X_{t}^{(1)}, \ldots, X_{t}^{(n)}\right)$,

for $n$ independent adapted stochastic processes $X_{t}^{(1)}, \ldots$, $X_{t}^{(n)}$, which represent the factors. To ensure that $S_{t}$ is adapted as well, we assume that he function $g: \mathbb{R}^{n} \rightarrow \mathbb{R}$ be continuous. The spot price model in (4) is very general and includes many interesting cases known in the energy and commodity markets. Here, we list a few examples and connect them to our model. Schwartz (1997) introduced a simple model for oil price dynamics called the Schwartz model, which is defined as follows:

$S_{t}=e^{U_{t}}$,

with

$\mathrm{d} U_{t}=\kappa\left(\theta-X_{t}\right) \mathrm{d} t+\sigma \mathrm{d} W_{t}$,

where $\theta>0$ is the mean-reverting level, $\kappa>0$ is the rate at which $U_{t}$ is pulled back to the level $\theta, \sigma>0$ is the volatility rate of $U_{t}$, and $W_{t}$ is a Brownian motion. After that, two-factor extension of the commodity Schwartz model is proposed by Schwartz and Smith (2000). It takes the form

$S_{t}=S_{0} \exp \left\{X_{t}+Y_{t}\right\}$,

with

$\mathrm{d} X_{t}=-\theta X_{t} \mathrm{~d} t+\sigma \mathrm{d} W_{t}^{(1)}$,

$\mathrm{d} Y_{t}=\mu \mathrm{d} t+\xi \mathrm{d} W_{t}^{(2)}$

where the short-run deviations (i.e., $X_{t}$ ) are assumed to revert toward zero following an Ornstein-Uhlenbeck process and the equilibrium level (i.e., $Y_{t}$ ) is a drifted Brownian motion. $W_{t}^{(1)}$ and $W_{t}^{(2)}$ are two Brownian motions with correlation $\rho \in(-1,1)$. Moreover, $\theta, \mu, \sigma, \xi$ are mean-reversion coefficient of short-term deviation, drift parameter of equilibrium level, volatility rate of short-term deviation and volatility rate of equilibrium level, respectively. We note that $\theta>0$ describes the rate at which the short-term deviations are expected to disappear.

Lucia and Schwartz (2002) by adding the seasonal factor to the commodity model (5) presented the dynamics of electricity spot price as follows:

$S_{t}=f_{t} \exp \left\{X_{t}+Y_{t}\right\}$

where $f_{t}, X_{t}$ and $Y_{t}$ are given by Eqs. (3), (6) and (7), respectively.
Following Benth et al. (2010), we consider the electricity spot price dynamic as follows:

$S(t)=f(t) \exp \left\{X_{t}+Y_{t}\right\}$

where

$$
\begin{aligned}
\mathrm{d} X_{t} & =\left(\kappa-\theta X_{t}\right) \mathrm{d} t+\sigma \mathrm{d} W_{t}^{(1)}, \\
\mathrm{d} Y_{t} & =\mu \mathrm{d} t+\xi \mathrm{d} W_{t}^{(2)},
\end{aligned}
$$

and the short-run deviations $X_{t}$ mean-revert toward a level given by the $\kappa$. We note that if $\kappa=0$, then model (9) becomes model (8).

A dynamics for the spot price evolution is desirable for several reasons. The models that describe the uncertainty of spot price are of interest to traders operating in the energy markets (see Benth et al. 2008). However, as stated in Benth et al. (2008), the uncertain models are also used as a reference point for the settlement of future and forward contracts and are therefore an essential input in understanding the dynamics of energy derivatives. In addition, since energy commodities are driven by the balance between demand and production, prices return to their mean levels. A natural class of stochastic models to describe such dynamics is the Ornstein-Uhlenbeck processes (see Benth et al. 2008). When we use the models based on the stochastic processes, which are defined on probability space, a large sample size is needed to estimate probability distribution based on long-run frequency (see Liu 2012a). However, Liu (2012a) stated that the sample size is often small (even no sample) in practice and the belief degree usually has much larger variance than the long-run frequency. Thus, we should deal with it by using uncertainty theory. Actually, we intend to express the electricity models in an uncertain space and create a connection between electricity markets and this space. If we assume that the electricity spot price follows some uncertain differential equation, then we may produce a new topic of uncertain electricity. Let the logarithmic electricity spot prices represent by $Z_{t}$ with $0 \leq t \leq T$, as the sum of three components with canonical processes. Which the first one is considered the logarithmic seasonality function and is represented by a known deterministic function of time, $F_{t}$. The second and third parts are the uncertain Ornstein-Uhlenbeck process $X_{t}$ and an ordinary uncertain differential equation $Y_{t}$. In this case, if Wiener processes $W_{t}^{(1)}$ and $W_{t}^{(2)}$ in Eqs. (10) and (11) are replaced by Liu processes $C_{t}^{(1)}$ and $C_{t}^{(2)}$, we have an electricity two-factor model in which the electricity spot price $S_{t}$ is determined by

$\left\{\begin{array}{l}S_{t}=f_{t} \exp \left\{X_{t}+Y_{t}\right\}, \\ \mathrm{d} X_{t}=\left(\kappa-\theta X_{t}\right) \mathrm{d} t+\sigma \mathrm{d} C_{t}^{(1)}, \quad X_{0}=0, \\ \mathrm{~d} Y_{t}=\mu \mathrm{d} t+\xi \mathrm{d} C_{t}^{(2)}, \quad Y_{0}=0,\end{array}\right.$ 
where $X_{t}$ and $Y_{t}$ are, respectively, short-term deviation and equilibrium level processes, which are driven by Liu processes. Moreover, $\theta, \mu, \sigma, \xi$ and $\kappa$ are mean-reversion coefficient of short-term deviation, drift parameter of equilibrium level, volatility rate of short-term deviation, volatility rate of equilibrium level and mean reversion of short-run deviations (i.e., the short-run deviations $X_{t}$ mean-revert toward a level given by the $\kappa$ ), respectively.

Since in real market, electricity spot prices return rates are more concerned, the logarithms of the electricity spot prices $Z_{t}, 0 \leq t \leq T$ are conducted as follows:

$\left\{\begin{array}{l}Z_{t}=F_{t}+X_{t}+Y_{t}, \\ \mathrm{~d} X_{t}=\left(\kappa-\theta X_{t}\right) \mathrm{d} t+\sigma \mathrm{d} C_{t}^{(1)}, \quad X_{0}=0, \\ \mathrm{~d} Y_{t}=\mu \mathrm{d} t+\xi \mathrm{d} C_{t}^{(2)}, \quad Y_{0}=0,\end{array}\right.$

where $F_{t}=\ln f_{t}$.

Theorem 6 The solution of the logarithmic uncertain electricity model $Z_{t}$ in system (13) is as follows:

$Z_{t}=F_{t}+X_{t}+Y_{t}$,

where

$X_{t}=\frac{\kappa}{\theta}\left(1-e^{-\theta t}\right)+\sigma e^{-\theta t} \int_{0}^{t} e^{\theta s} \mathrm{~d} C_{s}^{(1)}$,

$Y_{t}=\mu t+\xi C_{t}^{(2)}$.

Proof The proof follows easily from Chen and Liu (2010) and is omitted here.

At any given time $t$, the solutions $X_{t}$ and $Y_{t}$ in Theorem 6 are normal uncertain variables with expected values

$\frac{\kappa}{\theta}\left(1-e^{-\theta t}\right), \quad \mu t$

and standard deviations

$\frac{\sigma e^{-\theta t}}{\theta}\left(e^{\theta t}-1\right), \quad \xi t$

respectively. From Definition 5, $X_{t}$ and $Y_{t}$ can be expressed as follows:

$X_{t} \sim \mathcal{N}\left(\frac{\kappa}{\theta}\left(1-e^{-\theta t}\right), \frac{\sigma e^{-\theta t}}{\theta}\left(e^{\theta t}-1\right)\right), \quad Y_{t} \sim \mathcal{N}(\mu t, \xi t)$.

Thus, by applying Theorem 2 , the logarithmic electricity spot price $Z_{t}$ is a normal uncertain variable with expected value

$F_{t}+\frac{\kappa}{\theta}\left(1-e^{-\theta t}\right)+\mu t$ and standard deviation

$\frac{\sigma e^{-\theta t}}{\theta}\left(e^{\theta t}-1\right)+\xi t$

which is denoted as

$Z_{t} \sim \mathcal{N}\left(F_{t}+\frac{\kappa}{\theta}\left(1-e^{-\theta t}\right)+\mu t, \frac{\sigma e^{-\theta t}}{\theta}\left(e^{\theta t}-1\right)+\xi t\right)$.

The uncertainty distribution $\Phi_{t}$ of $Z_{t}$ at any time $t$ is

$\Phi_{t}(z)=\left(1+\exp \left\{\frac{\pi\left(\frac{\kappa}{\theta}\left(1-e^{-\theta t}\right)+\mu t+F_{t}-z\right)}{\sqrt{3}\left(\xi t+\frac{\sigma}{\theta}\left(1-e^{-\theta t}\right)\right)}\right\}\right)^{-1}$.

Before discussing the confidence interval and some other important properties of logarithmic electricity spot price $Z_{t}$, we prove that $Z_{t}$ satisfying in the Yao-Chen formula. The next theorem plays an important role in simulation of the electricity spot price.

Theorem 7 (Hassanzadeh and Mehrdoust 2020) Assume that for $i=1,2, f_{i}$ and $g_{i}$ are continuous functions and $C_{t}^{(1)}$ and $C_{t}^{(2)}$ are independent canonical Liu processes. Suppose that $Y_{i t}$ and $Y_{i t}^{\alpha}$ be the solution and $\alpha$-path of an uncertain differential equation

$\mathrm{d} Y_{i t}=f_{i}\left(t, Y_{i t}\right) \mathrm{d} t+g_{i}\left(t, Y_{i t}\right) \mathrm{d} C_{t}^{(i)}, \quad i=1,2$,

respectively. Let $\left|h_{i}(t, y)\right|$ be a continuous increasing function. Then, the solution $U_{t}$ of an uncertain differential equation

$\mathrm{d} U_{t}=f\left(t, U_{t}\right) \mathrm{d} t+\sum_{i=1}^{2} h_{i}\left(t, Y_{i t}\right) g_{i}\left(t, U_{t}\right) \mathrm{d} C_{t}^{(i)}$

is a contour process with an $\alpha$-path $U_{t}^{\alpha}$ that solves the corresponding ordinary differential equation

$\mathrm{d} U_{t}^{\alpha}=f\left(t, U_{t}^{\alpha}\right) \mathrm{d} t+\sum_{i=1}^{2}\left|h_{i}\left(t, Y_{i t}^{\alpha}\right) g_{i}\left(t, U_{t}^{\alpha}\right)\right| \Phi^{-1}(\alpha) \mathrm{d} t$,

where

$\Phi^{-1}(\alpha)=\frac{\sqrt{3}}{\pi} \ln \frac{\alpha}{1-\alpha}, \quad \alpha \in(0,1)$.

In other words,

$\mathcal{M}\left\{U_{t} \leq U_{t}^{\alpha}\right\}=\alpha, \quad \mathcal{M}\left\{U_{t}>U_{t}^{\alpha}\right\}=1-\alpha$. 
Corollary 1 The process followed by $Z_{t}$ can be expressed as the solution to the following stochastic differential equation (provided again that the function $F_{t}=\ln f_{t}$ satisfies appropriate regularity conditions)

$$
\begin{aligned}
\mathrm{d} Z_{t}= & \mathrm{d} F_{t}+\left(\kappa-\theta X_{t}\right) \mathrm{d} t+\mu \mathrm{d} t+\sigma \mathrm{d} C_{t}^{(1)}+\xi \mathrm{d} C_{t}^{(2)} \\
= & \left(F_{t}^{\prime}+\mu+\kappa+\theta F_{t}+\theta Y_{t}-\theta Z_{t}\right) \mathrm{d} t \\
& +\sigma \mathrm{d} C_{t}^{(1)}+\xi \mathrm{d} C_{t}^{(2)} \\
= & \left(F_{t}^{\prime}+\mu+\kappa+\theta F_{t}+\theta \mu t+\theta \xi C_{t}^{(2)}-\theta Z_{t}\right) \mathrm{d} t \\
& +\sigma \mathrm{d} C_{t}^{(1)}+\xi \mathrm{d} C_{t}^{(2)} .
\end{aligned}
$$

Now, we define

$f\left(t, Z_{t}\right)=F_{t}^{\prime}+\mu+\kappa+\theta F_{t}+\theta \mu t+\theta \xi C_{t}^{(2)}-\theta Z_{t}$,

$h_{1}\left(t, X_{t}\right)=h_{2}\left(t, Y_{t}\right)=1, \quad g_{1}\left(t, Z_{t}\right)=\xi, \quad g_{2}\left(t, Z_{t}\right)=\sigma$.

Then, we have

$$
\begin{aligned}
\mathrm{d} Z_{t}= & f\left(t, Z_{t}\right) \mathrm{d} t+h_{1}\left(t, X_{t}\right) g_{1}\left(t, Z_{t}\right) \mathrm{d} C_{t}^{(1)} \\
& +h_{2}\left(t, Y_{t}\right) g_{2}\left(t, Z_{t}\right) \mathrm{d} C_{t}^{(2)}
\end{aligned}
$$

From Theorem 7, the uncertain differential equation (19) is a contour process with an $\alpha$-path $Z_{t}^{\alpha}$ that solves the corresponding ordinary differential equation

$$
\begin{aligned}
\mathrm{d} Z_{t}^{\alpha}= & f\left(t, Z_{t}^{\alpha}\right) \mathrm{d} t+\left(h_{1}\left(t, X_{t}^{\alpha}\right) g_{1}\left(t, Z_{t}^{\alpha}\right)\right. \\
& \left.+h_{2}\left(t, Y_{t}^{\alpha}\right) g_{2}\left(t, Z_{t}^{\alpha}\right)\right) \Phi^{-1}(\alpha) \mathrm{d} t .
\end{aligned}
$$

In other words,

$\mathcal{M}\left\{Z_{t} \leq Z_{t}^{\alpha}\right\}=\alpha, \quad \mathcal{M}\left\{Z_{t}>Z_{t}^{\alpha}\right\}=1-\alpha$.

We note that

$\mathrm{d} Z_{t}^{\alpha}=\left(F_{t}^{\prime}+\mu+\kappa-\theta X_{t}^{\alpha}+\frac{\sqrt{3}}{\pi}(\sigma+\xi) \ln \frac{\alpha}{1-\alpha}\right) \mathrm{d} t$,

where

$X_{t}^{\alpha}=\frac{\left(\pi \kappa+\sqrt{3} \sigma \ln \frac{\alpha}{1-\alpha}\right)\left(1-e^{-\theta t}\right)}{\theta \pi}$.

Given a confidence level $\alpha(0<\alpha<1)$, the $\alpha$ confidence interval for $Z_{t}$ is suggested as

$$
\left[\mathbb{E}\left[Z_{t}\right]-\ell, \mathbb{E}\left[Z_{t}\right]+\ell\right]
$$

where $\ell$ is the solution of

$$
\Phi_{t}\left(\mathbb{E}\left[Z_{t}\right]+\ell\right)-\Phi_{t}\left(\mathbb{E}\left[Z_{t}\right]-\ell\right)=\alpha .
$$

Denote the uncertain measure as $\mathcal{M}$, which indicates the belief degree that an uncertain event may happen. It follows from the subadditivity axiom of uncertain measure in Definition 1 that

$$
\begin{aligned}
& \mathcal{M}\left\{\mathbb{E}\left[Z_{t}\right]-\ell<Z_{t}<\mathbb{E}\left[Z_{t}\right]+\ell\right\} \\
& \geq \Phi_{t}\left(\mathbb{E}\left[Z_{t}\right]+\ell\right)-\Phi_{t}\left(\mathbb{E}\left[Z_{t}\right]-\ell\right)=\alpha .
\end{aligned}
$$

Thus, we have the chance of $\alpha$ to cover the logarithmic electricity spot price $Z_{t}$ with this confidence interval.

Proposition 1 For the uncertain model (13), the $\alpha(0<\alpha<$ 1) confidence interval of the log-electricity spot price $Z_{t}$ at any time $t$ can be expressed as follows:

$$
\begin{gathered}
{\left[\frac{\kappa}{\theta}\left(1-e^{-\theta t}\right)+\mu t+F_{t}-\frac{\sqrt{3}}{\pi}\left(\xi t+\frac{\sigma}{\theta}-\frac{\sigma}{\theta} e^{-\theta t}\right) \ln \frac{1+\alpha}{1-\alpha},\right.} \\
\left.\frac{\kappa}{\theta}\left(1-e^{-\theta t}\right)+\mu t+F_{t}+\frac{\sqrt{3}}{\pi}\left(\xi t+\frac{\sigma}{\theta}-\frac{\sigma}{\theta} e^{-\theta t}\right) \ln \frac{1+\alpha}{1-\alpha}\right] .
\end{gathered}
$$

Proof We put

$\mathbb{E}\left[Z_{t}\right]=\bar{C}_{t}, \quad \Phi_{t}(z)=\left(1+\exp \left\{\frac{\pi\left(\bar{C}_{t}-z\right)}{\bar{D}_{t}}\right\}\right)^{-1}$,

and

$$
\begin{gathered}
\bar{C}_{t}=\frac{\kappa}{\theta}\left(1-e^{-\theta t}\right)+\mu t+F_{t}, \\
\bar{D}_{t}=\sqrt{3}\left(\xi t+\frac{\sigma}{\theta}-\frac{\sigma}{\theta} e^{-\theta t}\right) .
\end{gathered}
$$

We now obtain the value of $\ell$. From Eq. (22), we have

$$
\begin{aligned}
& \left(1+\exp \left\{\frac{\pi\left(\bar{C}_{t}-\bar{C}_{t}-\ell\right)}{\bar{D}_{t}}\right\}\right)^{-1} \\
& -\left(1+\exp \left\{\frac{\pi\left(\bar{C}_{t}-\bar{C}_{t}+\ell\right)}{\bar{D}_{t}}\right\}\right)^{-1}=\alpha .
\end{aligned}
$$

Thus,

$$
\left(1+\exp \left\{\frac{-\pi \ell}{\bar{D}_{t}}\right\}\right)^{-1}-\left(1+\exp \left\{\frac{\pi \ell}{\bar{D}_{t}}\right\}\right)^{-1}=\alpha,
$$

which implies

$$
\frac{\exp \left\{\frac{\pi \ell}{\bar{D}_{t}}\right\}-\exp \left\{\frac{-\pi \ell}{\bar{D}_{t}}\right\}}{2+\exp \left\{\frac{\pi \ell}{\bar{D}_{t}}\right\}+\exp \left\{\frac{-\pi \ell}{\bar{D}_{t}}\right\}}=\alpha .
$$

Hence, by change of variable $U=\exp \left\{\frac{\pi \ell}{\bar{D}_{t}}\right\}$, we have

$$
\frac{U-U^{-1}}{2+U+U^{-1}}=\frac{U^{2}-1}{(U+1)^{2}}=\alpha .
$$


Thus, $U=\frac{1+\alpha}{1-\alpha}$, and we obtain

$\ell=\frac{1}{\pi} \bar{D}_{t} \ln U=\frac{1}{\pi} \bar{D}_{t} \ln \frac{1+\alpha}{1-\alpha}$.

Substituting the solution of $\ell$ into Eq. (21) proves the result.

As pointed out by Byström (2003), to update the hedge based on the dynamics model, future contracts on the Nordic energy exchange must be bought or sold every day. When traders' assets are about to be lost, they usually prefer to make a profit rather than make a loss. On the other hand, since the evolution of future contracts depend on the starting level of the spot price (see Cartea et al. 2005), many traders set a certain level to sell their future contracts if the electricity spot price reaches this level, with this approach they can maintain their profits. They can also set a limit for electricity spot price to buy future contracts. Due to the fact that logarithm is a strictly monotone increasing function, if the logarithmic electricity spot price reaches a level at time $t$, one can adopt the appropriate strategy for the future contract at time $t$. Theorems 8 and 9 state, respectively, the uncertain measure of logarithmic electricity spot price (i.e., $\left.\mathcal{M}\left(Z_{t} \geq z_{0}\right), z_{0} \in \mathbb{R}\right)$ and the uncertainty distribution of the first time that the logarithmic electricity spot price reaches a certain level $\eta$. From Liu (2007), we note that the uncertain measure $\mathcal{M}\left(Z_{t} \geq z_{0}\right)$ indicates the belief degree of an uncertain event $Z_{t} \geq z_{0}$.

Theorem 8 Consider the logarithmic uncertain electricity model $Z_{t}$ in system (13). Denoting the $M E V, z_{0}$, the uncertain measure for at time $t$ is as follows:

$$
\begin{aligned}
& \mathcal{M}\left(Z_{t} \geq z_{0}\right) \\
& =\left(1+\exp \left\{\frac{-\pi\left(\frac{\kappa}{\theta}\left(1-e^{-\theta t}\right)+\mu t+F_{t}-z_{0}\right)}{\sqrt{3}\left(\xi t+\frac{\sigma}{\theta}\left(1-e^{-\theta t}\right)\right)}\right\}\right)^{-1} .
\end{aligned}
$$

Proof It follows from Eq. (17) and the duality of the uncertain measure in Definition 1.

$$
\begin{aligned}
\mathcal{M} & \left(Z_{t} \geq z_{0}\right) \\
& =1-\Phi_{t}\left(z_{0}\right) \\
& =1-\left(1+\exp \left\{\frac{\pi\left(\frac{\kappa}{\theta}\left(1-e^{-\theta t}\right)+\mu t+F_{t}-z_{0}\right)}{\sqrt{3}\left(\xi t+\frac{\sigma}{\theta}\left(1-e^{-\theta t}\right)\right)}\right\}\right)^{-1} \\
& =\left(1+\exp \left\{\frac{-\pi\left(\frac{\kappa}{\theta}\left(1-e^{-\theta t}\right)+\mu t+F_{t}-z_{0}\right)}{\sqrt{3}\left(\xi t+\frac{\sigma}{\theta}\left(1-e^{-\theta t}\right)\right)}\right\}\right)^{-1} .
\end{aligned}
$$

For a given constant $\eta \in \mathbb{R}$, define the uncertain variable

$t_{\eta}=\inf \left\{t \geq 0 \mid Z_{t} \geq \eta\right\}$
Theorem 9 For a given constant $\eta \in \mathbb{R}$ and the logarithmic uncertain electricity model $Z_{t}$ in system (13), the level-time defined in (25) has an uncertainty distribution

$\Psi_{\eta}\left(t^{*}\right)=\left(1+\exp \left\{\frac{-\pi\left(\frac{\kappa}{\theta}\left(1-e^{-\theta t^{*}}\right)+\mu t^{*}+F_{t^{*}}-\eta\right)}{\sqrt{3}\left(\xi t^{*}+\frac{\sigma}{\theta}\left(1-e^{-\theta t^{*}}\right)\right)}\right\}\right)^{-1}$.

\section{Proof Denote}

$\alpha_{0}=\inf \left\{\alpha \mid \sup _{0 \leq t \leq t^{*}} \Phi_{t}^{-1}(\alpha) \geq \eta\right\}$

where

$$
\begin{aligned}
\Phi_{t}^{-1}(\alpha)= & \frac{\kappa}{\theta}\left(1-e^{-\theta t}\right)+\mu t+F_{t} \\
& +\frac{\sqrt{3}\left(\xi \theta t+\sigma-\sigma e^{-\theta t}\right)}{\pi \theta} \ln \frac{\alpha}{1-\alpha}
\end{aligned}
$$

is the inverse uncertainty distribution of the $Z_{t}$. It follows from definition of the level-time defined in (25) and $Z_{t}$ that

$$
\left\{Z_{t} \geq \Phi_{t}^{-1}\left(\alpha_{0}\right), \forall t\right\} \subset\left\{\sup _{0 \leq t \leq t^{*}} Z_{t} \geq \eta\right\}=\left\{t_{\eta} \leq t^{*}\right\}
$$

and

$$
\left\{Z_{t}<\Phi_{t}^{-1}\left(\alpha_{0}\right), \forall t\right\} \subset\left\{\sup _{0 \leq t \leq t^{*}} Z_{t}<\eta\right\}=\left\{t_{\eta}>t^{*}\right\}
$$

Using Yao-Chen formula, we have

$$
\mathcal{M}\left\{t_{\eta} \leq t^{*}\right\} \geq \mathcal{M}\left\{Z_{t} \geq \Phi_{t}^{-1}\left(\alpha_{0}\right)\right\}=1-\alpha_{0}
$$

and

$\mathcal{M}\left\{t_{\eta}>t^{*}\right\} \leq \mathcal{M}\left\{Z_{t}<\Phi_{t}^{-1}\left(\alpha_{0}\right)\right\}=\alpha_{0}$.

From the duality axiom in Definition 1 for the uncertain measure, we have

$\mathcal{M}\left\{t_{\eta} \leq t^{*}\right\}=1-\mathcal{M}\left\{t_{\eta}>t^{*}\right\} \leq 1-\alpha_{0}$.

Following inequalities (28) and (29), we obtain

$\Psi_{\eta}(z)=\mathcal{M}\left\{t_{\eta} \leq t^{*}\right\}=1-\alpha_{0}$

Notice that $\Phi_{t}^{-1}(\alpha)$ is a monotone function with respect to $t$. Thus,

$\left\{\sup _{0 \leq t \leq t^{*}} \Phi_{t}^{-1}(\alpha) \geq \eta\right\}=\left\{\Phi_{t^{*}}^{-1}(\alpha) \geq \eta\right\}$ 
From expression (27), we have

$$
\begin{aligned}
\alpha_{0} & =\inf \left\{\alpha \mid \sup _{0 \leq t \leq t^{*}} \Phi_{t}^{-1}(\alpha) \geq \eta\right\} \\
& =\inf \left\{\alpha \mid \Phi_{t^{*}}^{-1}(\alpha) \geq \eta\right\}=\inf \left\{\alpha \mid \alpha \geq \Phi_{t^{*}}(\eta)\right\}=\Phi_{t^{*}}(\eta) .
\end{aligned}
$$

As a result, we obtain

$\alpha_{0}=\left(1+\exp \left\{\frac{\pi\left(\frac{\kappa}{\theta}\left(1-e^{-\theta t^{*}}\right)+\mu t^{*}+F_{t^{*}}-\eta\right)}{\sqrt{3}\left(\xi t^{*}+\frac{\sigma}{\theta}\left(1-e^{-\theta t^{*}}\right)\right)}\right\}\right)^{-1}$.

Therefore,

$$
\begin{aligned}
& \Psi_{\eta}(z)=1-\alpha_{0} \\
& \quad=\left(1+\exp \left\{\frac{-\pi\left(\frac{\kappa}{\theta}\left(1-e^{-\theta t^{*}}\right)+\mu t^{*}+F_{t^{*}}-\eta\right)}{\sqrt{3}\left(\xi t^{*}+\frac{\sigma}{\theta}\left(1-e^{-\theta t^{*}}\right)\right)}\right\}\right)^{-1},
\end{aligned}
$$

and the proof is completed.

In the electricity exchange market, trading the future and forward contracts is based on the spot prices. The main difference between such contracts and other products in commodity markets is that in electricity markets, contracts are delivered over a period, not at a specific time. In the electricity market, these products are cash settled based on the spot price in the settlement period (see Benth et al. 2008). From Benth et al. (2007), the electricity is delivered as a flow of rate $Z(t) / T, 0 \leq t \leq T$ during the settlement period [0,T], giving a total cost (in logarithmic units) of

$G_{T}:=\frac{1}{T} \int_{0}^{T} Z_{S} \mathrm{~d} s$

The following two theorems express the expected value and confidence interval for $G_{t}=\frac{1}{T} \int_{0}^{t} Z_{s} \mathrm{~d} s$.

Theorem 10 For the uncertain model in (13), the $G_{t}$ has the inverse uncertainty distribution

$$
\begin{aligned}
\Upsilon_{t}^{-1}(\alpha)= & \frac{1}{T}\left(\frac{\kappa}{\theta}\left(t+\frac{e^{-\theta t}-1}{\theta}\right)+\frac{\mu t^{2}}{2}+\mathbf{F}_{t}\right. \\
& \left.+\frac{\sqrt{3}}{\pi \theta} \ln \frac{\alpha}{1-\alpha}\left(\frac{\xi \theta t^{2}}{2}+\sigma t+\frac{\sigma}{\theta}\left(e^{-\theta t}-1\right)\right)\right),
\end{aligned}
$$

where

$\mathbf{F}_{t}=\int_{0}^{t} \ln \left(a+b s+c \sin \left(\frac{2 \pi(s-d)}{365}\right)\right) \mathrm{d} s$.

Moreover, the expected value is

$\mathbb{E}\left[G_{t}\right]=\frac{1}{T}\left(\frac{\kappa}{\theta}\left(t+\frac{e^{-\theta t}-1}{\theta}\right)+\frac{\mu t^{2}}{2}+\mathbf{F}_{t}\right)$.
Proof For a fixed maturity time $T>0$, the product of a normal uncertain variable $Z_{t}$ in Eq. (16) and the scalar number $1 / T$ is also a normal uncertain variable with expectation

$\frac{1}{T}\left(F_{t}+\frac{\kappa}{\theta}\left(1-e^{-\theta t}\right)+\mu t\right)$

and standard deviation

$\frac{1}{T}\left(\frac{\sigma e^{-\theta t}}{\theta}\left(e^{\theta t}-1\right)+\xi t\right)$

Thus, the uncertainty distribution $\varpi_{t}$ of $\frac{1}{T} Z_{t}$ at any time $t$ is as follows:

$\varpi_{t}(z)=\left(1+\exp \left\{\frac{\pi\left(\frac{\kappa}{\theta}\left(1-e^{-\theta t}\right)+\mu t+F_{t}-z T\right)}{\sqrt{3}\left(\xi t+\frac{\sigma}{\theta}\left(1-e^{-\theta t}\right)\right)}\right\}\right)^{-1}$.

Moreover, $\frac{1}{T} Z_{t}$ has the inverse uncertainty distribution as follows:

$$
\begin{aligned}
\varpi_{t}^{-1}(\alpha)= & \frac{1}{T}\left(\frac{\kappa}{\theta}\left(1-e^{-\theta t}\right)+\mu t+F_{t}\right. \\
& \left.+\frac{\sqrt{3}\left(\xi \theta t+\sigma-\sigma e^{-\theta t}\right)}{\pi \theta} \ln \frac{\alpha}{1-\alpha}\right) .
\end{aligned}
$$

Obviously, for any time $0 \leq t \leq T$, we have

$\left\{\frac{1}{T} Z_{s} \leq \varpi_{s}^{-1}(\alpha), \forall s\right\} \subset\left\{\int_{0}^{t} \frac{1}{T} Z_{s} \mathrm{~d} s \leq \int_{0}^{t} \varpi_{s}^{-1}(\alpha) \mathrm{d} s\right\}$

and

$\left\{\frac{1}{T} Z_{s}>\varpi_{s}^{-1}(\alpha), \forall s\right\} \subset\left\{\int_{0}^{t} \frac{1}{T} Z_{s} \mathrm{~d} s>\int_{0}^{t} \varpi_{s}^{-1}(\alpha) \mathrm{d} s\right\}$.

Using Yao-Chen formula, we have

$$
\begin{aligned}
& \mathcal{M}\left\{\int_{0}^{t} \frac{1}{T} Z_{s} \mathrm{~d} s \leq \int_{0}^{t} \varpi_{s}^{-1}(\alpha) \mathrm{d} s\right\} \\
& \geq \mathcal{M}\left\{\frac{1}{T} Z_{s} \leq \varpi_{s}^{-1}(\alpha), \forall s\right\}=\alpha
\end{aligned}
$$

and

$$
\begin{aligned}
& \mathcal{M}\left\{\int_{0}^{t} \frac{1}{T} Z_{s} \mathrm{~d} s>\int_{0}^{t} \varpi_{s}^{-1}(\alpha) \mathrm{d} s\right\} \\
& \geq \mathcal{M}\left\{\frac{1}{T} Z_{s}>\varpi_{s}^{-1}(\alpha), \forall s\right\}=1-\alpha .
\end{aligned}
$$

From the duality axiom for the uncertainty measure in Definition 1 , we have

$\mathcal{M}\left\{\int_{0}^{t} \frac{1}{T} Z_{s} \mathrm{~d} s \leq \int_{0}^{t} \varpi_{s}^{-1}(\alpha) \mathrm{d} s\right\}$ 


$$
\begin{aligned}
& =1-\mathcal{M}\left\{\int_{0}^{t} \frac{1}{T} Z_{s} \mathrm{~d} s \geq \int_{0}^{t} \varpi_{s}^{-1}(\alpha) \mathrm{d} s\right\} \leq 1-(1-\alpha) \\
= & \alpha .
\end{aligned}
$$

From inequalities (33) and (35), we obtain

$$
\mathcal{M}\left\{\frac{1}{T} \int_{0}^{t} Z_{s} \mathrm{~d} s \leq \int_{0}^{t} \varpi_{s}^{-1}(\alpha) \mathrm{d} s\right\}=\alpha .
$$

Thus, $G_{t}=\frac{1}{T} \int_{0}^{t} Z_{s} \mathrm{~d} s$ has the inverse uncertainty distribution as follows:

$$
\begin{aligned}
\Upsilon_{t}^{-1}(\alpha)= & \frac{1}{T} \int_{0}^{t} \varpi_{s}^{-1}(\alpha) \mathrm{d} s \\
= & \frac{1}{T} \int_{0}^{t}\left[\frac{\kappa}{\theta}\left(1-e^{-\theta s}\right)+\mu s+F_{s}\right. \\
& \left.+\frac{\sqrt{3}\left(\xi \theta s+\sigma-\sigma e^{-\theta s}\right)}{\pi \theta} \ln \frac{\alpha}{1-\alpha}\right] \mathrm{d} s \\
= & \frac{1}{T}\left(\frac{\kappa}{\theta}\left(t+\frac{e^{-\theta t}-1}{\theta}\right)+\frac{\mu t^{2}}{2}+\mathbf{F}_{t}\right. \\
& \left.+\frac{\sqrt{3}}{\pi \theta} \ln \frac{\alpha}{1-\alpha}\left(\frac{\xi \theta t^{2}}{2}+\sigma t+\frac{\sigma}{\theta}\left(e^{-\theta t}-1\right)\right)\right) .
\end{aligned}
$$

Due to Theorem 3, the expected value of $G_{t}$ can be calculated as follows:

$$
\begin{aligned}
\mathbb{E}\left[G_{t}\right] & =\int_{0}^{1} \Upsilon_{t}^{-1}(\alpha) \mathrm{d} \alpha \\
& =\frac{1}{T}\left(\frac{\kappa}{\theta}\left(t+\frac{e^{-\theta t}-1}{\theta}\right)+\frac{\mu t^{2}}{2}+\mathbf{F}_{t}\right),
\end{aligned}
$$

and the proof is completed.

Theorem 11 For the uncertain model in (13), the $G_{t}$ has an $\alpha(0<\alpha<1)$ confidence interval

$$
\left[\mathbb{E}\left[G_{t}\right]-\lambda^{*}, \quad \mathbb{E}\left[G_{t}\right]+\lambda^{*}\right],
$$

where

$$
\begin{aligned}
\lambda^{*} & =\inf \{\lambda>0 \mid H(\lambda) \geq \alpha\}, \\
H(\lambda) & =\Upsilon_{t}\left(\mathbb{E}\left[G_{t}\right]+\lambda\right)-\Upsilon_{t}\left(\mathbb{E}\left[G_{t}\right]-\lambda\right)
\end{aligned}
$$

and $\Upsilon_{t}$ is the uncertainty distribution for $G_{t}$.

Proof It follows from Theorem 1

$$
\begin{aligned}
& \mathcal{M}\left\{\mathbb{E}\left[G_{t}\right]-\lambda \leq G_{t} \leq \mathbb{E}\left[G_{t}\right]+\lambda\right\} \\
& \quad \geq \mathcal{M}\left\{G_{t} \leq \mathbb{E}\left[G_{t}\right]+\lambda\right\}-\mathcal{M}\left\{G_{t} \leq \mathbb{E}\left[G_{t}\right]-\lambda\right\} \\
& \quad=\Upsilon_{t}\left(\mathbb{E}\left[G_{t}\right]+\lambda\right)-\Upsilon_{t}\left(\mathbb{E}\left[G_{t}\right]-\lambda\right) .
\end{aligned}
$$

Due to the fact that $0<\Upsilon_{t}\left(\mathbb{E}\left[G_{t}\right]-\lambda\right)<1$, and by applying Yao-Chen formula, we conclude that

$\mathcal{M}\left\{\mathbb{E}\left[G_{t}\right]-\lambda \leq G_{t} \leq \mathbb{E}\left[G_{t}\right]+\lambda\right\} \geq \Upsilon_{t}\left(\mathbb{E}\left[G_{t}\right]+\lambda\right) \geq \alpha$.

Thus, we have the chance of $\alpha$ to cover $G_{t}$ with this confidence interval and the theorem follows immediately.

\section{Framework of calibration}

To compare simulated data with real market data, estimation of the parameters $\kappa, \theta, \mu, \sigma$ and $\xi$ in the logarithmic electricity model based on the observations is a crucial problem. Since the moment estimations for these parameters are not exist, in this section we derive generalized moment estimations for unknown parameters $\kappa, \theta, \mu, \sigma$ and $\xi$ in the logarithmic electricity spot model in Theorem 6.

Theorem 12 Consider the logarithmic electricity model in Theorem 6 with unknown parameters $\kappa, \theta, \mu, \sigma$ and $\xi$. Assume that there are $n$ observations $z_{t_{1}}, z_{t_{2}}, \ldots, z_{t_{n}}$ of the log-spot price of electricity $Z_{t}$ at time $t_{1}, t_{2}, \ldots, t_{n}$ with $0<t_{1}<t_{2}<\cdots<t_{n}$, respectively. The corresponding generalized moment estimation $\left(\kappa^{*}, \theta^{*}, \mu^{*}, \sigma^{*}, \xi^{*}\right)$ is the optimal solution of

$\min _{\kappa^{*}, \theta^{*}, \mu^{*}, \sigma^{*}, \xi^{*}} \sum_{k=1}^{5}\left(\frac{1}{n-1} \sum_{i=1}^{n-1}\left(\Xi_{i}\left(\kappa^{*}, \theta^{*}, \mu^{*}, \sigma^{*}, \xi^{*}\right)\right)^{k}-\beta_{k}\right)^{2}$,

where

$$
\begin{aligned}
& \Xi_{i}(\kappa, \theta, \mu, \sigma, \xi) \\
& =\frac{z_{t_{i+1}}-z_{t_{i}}-\left(F_{t_{i}}^{\prime}+\mu+\kappa+\theta F_{t_{i}}+\theta \mu t_{i}-\theta z_{t_{i}}\right)\left(t_{i+1}-t_{i}\right)}{\left(t_{i+1}-t_{i}\right)\left(\sigma+\xi+\theta \xi t_{i}\right)},
\end{aligned}
$$

and

$\beta_{k}=\left(\frac{1+(-1)^{k}}{2}\right)\left(\frac{21}{5}\right)^{\frac{k-2}{2}}, \quad k=1, \ldots, 5$.

Proof Equation (18) has a difference form

$$
\begin{aligned}
Z_{t_{i+1}=}= & Z_{t_{i}}+\left(F_{t_{i}}^{\prime}+\mu+\kappa+\theta F_{t_{i}}+\theta \mu t_{i}-\theta Z_{t_{i}}\right)\left(t_{i+1}-t_{i}\right) \\
& +\sigma\left(C_{t_{i+1}}^{(1)}-C_{t_{i}}^{(1)}\right)+\xi\left(C_{t_{i+1}}^{(2)}-C_{t_{i}}^{(2)}\right)+\theta \xi\left(t_{i+1}-t_{i}\right) C_{t_{i}}^{(2)} .
\end{aligned}
$$

On the other hand,

$$
\begin{aligned}
& \left(C_{t_{i+1}}^{(1)}-C_{t_{i}}^{(1)}\right) \sim \mathcal{N}\left(0, t_{i+1}-t_{i}\right), \\
& \left(C_{t_{i+1}}^{(2)}-C_{t_{i}}^{(2)}\right) \sim \mathcal{N}\left(0, t_{i+1}-t_{i}\right),
\end{aligned}
$$




$$
C_{t_{i}}^{(2)} \sim \mathcal{N}\left(0, t_{i}\right)
$$

Due to the fact that $\left(C_{t_{i+1}}^{(1)}-C_{t_{i}}^{(1)}\right), C_{t_{i+1}}^{(2)}-C_{t_{i}}^{(2)}$ and $C_{t_{i}}^{(2)}$ are independent, we have

$$
\begin{aligned}
& \left(\sigma\left(C_{t_{i+1}}^{(1)}-C_{t_{i}}^{(1)}\right)+\xi\left(C_{t_{i+1}}^{(2)}-C_{t_{i}}^{(2)}\right)+\theta \xi\left(t_{i+1}-t_{i}\right) C_{t_{i}}^{(2)}\right) \\
& \quad \sim \mathcal{N}\left(0,\left(t_{i+1}-t_{i}\right)\left(\sigma+\xi+\theta \xi t_{i}\right)\right) .
\end{aligned}
$$

Based on Definition 8 of Liu process, the following expression

$$
\begin{aligned}
& \frac{\left(\sigma\left(C_{t_{i+1}}^{(1)}-C_{t_{i}}^{(1)}\right)+\xi\left(C_{t_{i+1}}^{(2)}-C_{t_{i}}^{(2)}\right)+\theta \xi\left(t_{i+1}-t_{i}\right) C_{t_{i}}^{(2)}\right)}{\left(t_{i+1}-t_{i}\right)\left(\sigma+\xi+\theta \xi t_{i}\right)} \\
& \quad \sim \mathcal{N}(0,1)
\end{aligned}
$$

is a standard normal uncertain variable with expected value 0 and variance 1 , which has an uncertainty distribution

$\Phi(x)=\left(1+\exp \left(-\frac{\pi x}{\sqrt{3}}\right)\right)^{-1}$.

From Eq. (38), we have

$$
\begin{aligned}
& Z_{t_{i+1}}-Z_{t_{i}}-\left(F_{t_{i}}^{\prime}+\mu+\kappa+\theta F_{t_{i}}+\theta \mu t-\theta Z_{t_{i}}\right)\left(t_{i+1}-t_{i}\right) \\
& =\left(\sigma\left(C_{t_{i+1}}^{(1)}-C_{t_{i}}^{(1)}\right)+\xi\left(C_{t_{i+1}}^{(2)}-C_{t_{i}}^{(2)}\right)+\theta \xi\left(t_{i+1}-t_{i}\right) C_{t_{i}}^{(2)}\right) .
\end{aligned}
$$

Therefore, the estimation of the parameters $\kappa, \theta, \mu, \sigma$ and $\xi$ is assumed to follow the standard normal uncertainty distribution, i.e.,

$\frac{Z_{t_{i+1}}-Z_{t_{i}}-\left(F_{t_{i}}^{\prime}+\mu+\kappa+\theta F_{t_{i}}+\theta \mu t_{i}-\theta Z_{t_{i}}\right)\left(t_{i+1}-t_{i}\right)}{\left(t_{i+1}-t_{i}\right)\left(\sigma+\xi+\theta \xi t_{i}\right)}$

$\sim \mathcal{N}(0,1)$.

Assume that there are $n$ observations $z_{1}, z_{2}, \ldots, z_{n}$ of the $\log$ energy spot price at the times $t_{1}, t_{2}, \ldots, t_{n}$ with $t_{1}<t_{2}<$ $\ldots<t_{n}$, respectively. Substituting $Z_{t_{i+1}}$ and $Z_{t_{i}}$ with the observations $z_{t_{i+1}}$ and $z_{t_{i}}$ in Eq. (39), we write

$$
\begin{aligned}
& \Xi_{i}(\kappa, \theta, \mu, \sigma, \xi) \\
& =\frac{z_{t_{i+1}}-z_{t_{i}}-\left(F_{t_{i}}^{\prime}+\mu+\kappa+\theta F_{t_{i}}+\theta \mu t_{i}-\theta z_{t_{i}}\right)\left(t_{i+1}-t_{i}\right)}{\left(t_{i+1}-t_{i}\right)\left(\sigma+\xi+\theta \xi t_{i}\right)} \\
& \quad \sim \mathcal{N}(0,1), \quad i=1,2, \ldots, n-1 .
\end{aligned}
$$

Note that $\Xi_{i}, i=1,2, \ldots, n-1$, are real functions of the parameters $\kappa, \theta, \mu, \sigma$ and $\xi$. For the estimates of $\kappa, \theta, \mu, \sigma$ and $\xi$ denoted by $\kappa^{*}, \theta^{*}, \mu^{*}, \sigma^{*}$ and $\xi^{*}$, it follows from Eq. (39) that the values of these functions

$$
\Xi_{1}\left(\kappa^{*}, \theta^{*}, \mu^{*}, \sigma^{*}, \xi^{*}\right), \Xi_{2}\left(\kappa^{*}, \theta^{*}, \mu^{*}, \sigma^{*}, \xi^{*}\right), \ldots,
$$

$$
\Xi_{n-1}\left(\kappa^{*}, \theta^{*}, \mu^{*}, \sigma^{*}, \xi^{*}\right)
$$

can be regarded as $n-1$ samples of a standard normal uncertainty distribution $\mathcal{N}(0,1)$. The sample moments would provide good estimates of the corresponding population moments. Further, the $k$ th sample moments are

$$
\frac{1}{n-1} \sum_{i=1}^{n-1}\left(\Xi_{i}\left(\kappa^{*}, \theta^{*}, \mu^{*}, \sigma^{*}, \xi^{*}\right)\right)^{k}, \quad k=1,2, \ldots, 5,
$$

and the $k$ th population moments are

$\beta_{k}=\left(\frac{\sqrt{3}}{\pi}\right)^{k} \int_{0}^{1}\left(\ln \frac{\alpha}{1-\alpha}\right)^{k} \mathrm{~d} \alpha, \quad k=1,2, \ldots, 5$.

We have $\beta_{k}=0$ for any positive odd number $k$, and

$\beta_{2}=1, \quad \beta_{4}=\frac{21}{5}$.

Specially, for $k=1,2, \ldots, 5$, we have

$\beta_{k}=\left(\frac{1+(-1)^{k}}{2}\right)\left(\frac{21}{5}\right)^{\frac{k-2}{2}}$.

Thus, the generalized moment estimation $\left(\kappa^{*}, \theta^{*}, \mu^{*}, \sigma^{*}, \xi^{*}\right)$ is the optimal solution of

$\min _{\kappa^{*}, \theta^{*}, \mu^{*}, \sigma^{*}, \xi^{*}} \sum_{k=1}^{5}\left(\frac{1}{n-1} \sum_{i=1}^{n-1}\left(\Xi_{i}\left(\kappa^{*}, \theta^{*}, \mu^{*}, \sigma^{*}, \xi^{*}\right)\right)^{k}-\beta_{k}\right)^{2}$.

Therefore, the result follows.

We use the $g a$ command of MATLAB software to solve the optimization problem (37). Genetic algorithm (GA) is a model of biological evolution based on Charles Darwin's theory of natural selection. Population size, selection rate, crossover and mutation probability, number of generations, convergence conditions, fit function (loss) and search space operators organize the essential part of the genetic algorithm as a problem-solving strategy. GA was first introduced by Holland (1975).

\section{Numerical results}

In this section, we present the theories expressed for the electricity spot price as numerically by using the electricity data set from the Nordic region of Oslo and Stockholm. We also compare the electricity spot price obtained by the uncertain two-factor model and the one-factor uncertainty model with the actual market data and show that the introduced twofactor model performs better. 
The electricity spot price $S_{t}^{o}$ under one-factor uncertainty model is expressed as follows:

$S_{t}^{o}=f_{t} e^{X_{t}}$

where

$$
\begin{aligned}
f_{t} & =a+b t+c \sin \left(\frac{2 \pi(t-d)}{365}\right), \\
\mathrm{d} X_{t} & =\left(\kappa-\theta X_{t}\right) \mathrm{d} t+\sigma \mathrm{d} C_{t},
\end{aligned}
$$

and $\mathrm{d} C_{t}$ is the increment of the Liu process and $a, b, c, d, \kappa, \theta$ $\in \mathbb{R}, \sigma>0$ are constants.

Suppose that $Z_{t}^{o}$ and $Z_{t}^{\alpha^{o}}$ be the solution and $\alpha$-path for logarithmic spot price of electricity under one-factor uncertainty model, respectively. Then, the solution $Z_{t}$ is a contour process with an $\alpha$-path $Z_{t}^{\alpha^{o}}$ that solves the corresponding ordinary differential equation

$$
\begin{aligned}
\mathrm{d} Z_{t}^{\alpha^{o}}= & \left(\kappa+\theta \ln \left(a+b t+c \sin \left(\frac{2 \pi(t-d)}{365}\right)\right)\right. \\
& +\frac{b+\frac{2 \pi c}{365} \cos \left(\frac{2 \pi(t-d)}{365}\right)}{a+b t+c \sin \left(\frac{2 \pi(t-d)}{365}\right)} \\
& \left.+\frac{\sqrt{3} \sigma}{\pi} \ln \frac{\alpha}{1-\alpha}-\theta Z_{t}^{\alpha^{o}}\right) \mathrm{d} t .
\end{aligned}
$$

In other words,

$$
\begin{aligned}
& \mathcal{M}\left\{Z_{t}^{o} \leq Z_{t}^{\alpha^{o}}, \forall t\right\}=\alpha, \\
& \mathcal{M}\left\{Z_{t}^{o}>Z_{t}^{\alpha^{o}}, \forall t\right\}=1-\alpha .
\end{aligned}
$$

Consider the logarithmic electricity model in system (40) with unknown parameters $\kappa, \theta$ and $\sigma$. Assume that there are $n$ observations $z_{t_{1}}, z_{t_{2}}, \ldots, z_{t_{n}}$ of the log-spot price of electricity $Z_{t}^{o}$ at time $t_{1}, t_{2}, \ldots, t_{n}$ with $0<t_{1}<t_{2}<\cdots<$ $t_{n}$, respectively. Similar to Theorem 12, the corresponding generalized moment estimation $\left(\kappa^{*}, \theta^{*}, \sigma^{*}\right)$ is the optimal solution of

$$
\min _{\kappa^{*}, \theta^{*}, \sigma^{*}} \sum_{k=1}^{3}\left(\frac{1}{n-1} \sum_{i=1}^{n-1}\left(\Xi_{i}\left(\kappa^{*}, \theta^{*} \sigma^{*}\right)\right)^{k}-\beta_{k}\right)^{2},
$$

where

$$
\begin{aligned}
& \Xi_{i}(\kappa, \theta, \mu, \sigma, \xi) \\
& =\frac{z_{t_{i+1}}-z_{t_{i}}-\left(F_{t_{i}}^{\prime}+\kappa+\theta F_{t_{i}}-\theta z_{t_{i}}\right)\left(t_{i+1}-t_{i}\right)}{\sigma\left(t_{i+1}-t_{i}\right)},
\end{aligned}
$$

and

$$
F_{t}=\ln f_{t}, \quad \beta_{k}=\left(\frac{1+(-1)^{k}}{2}\right)\left(\frac{21}{5}\right)^{\frac{k-2}{2}}, \quad k=1,2,3,
$$

The parameters of the seasonality function were estimated using the least squares approach. For this purpose, we have applied the nlinfit procedure in MATLAB software. We consider the estimated parameters as $a^{*}, b^{*}, c^{*}$ and $d^{*}$. All four parameters are significant at the $5 \%$ level, indicating that there are both significant seasonal variations and increase in electricity spot prices over the considered period. The electricity spot prices of the Nordic related to the Stockholm and Oslo regions with their seasonality function are graphed in Fig. 1. The estimated parameters of seasonality function $f_{t}$ are reported in Table 1.

Tables 2 and 3 report the estimated parameters of the uncertain two-factor model and the uncertain one-factor model related to the electricity spot prices of Stockholm and Oslo, respectively. The performance of the these models for the electricity spot price related to Stockholm and Oslo regions is represented in Table 4 for different horizons based on the the mean-absolute-error (MAE) criteria, respectively. As it can be readily seen from the table, our proposed uncertain two-factor model has a better performance in all time horizons. A close look at this table shows that the uncertain two-factor model has smaller MAE values than the other model considered for all horizons. Notice that the MAE is calculated as follows:

MAE $=\frac{1}{n} \sum_{i=1}^{n}\left|S_{t_{i}}^{*}-S_{t_{i}}\right|$

where $n$ is the total number of the electricity spot price data set, $S_{t_{i}}$ is the simulated electricity spot price and $S_{t_{i}}^{*}$ is the actual electricity spot price. It should be noted that to provide the numerical results, all the parameters of the proposed model are taken from Tables 1 and 2 .

From Eq. (14) and Proposition 1 in Sect. 3, the expected value and the $95 \%$ confidence interval for the logarithmic electricity spot price $Z_{t}$ are

$\mathbb{E}\left[Z_{t}\right]=F_{t}+\frac{\kappa}{\theta}\left(1-e^{-\theta t}\right)+\mu t$,

and

$$
\begin{aligned}
& {\left[\frac{\kappa}{\theta}\left(1-e^{-\theta t}\right)+\mu t+F_{t}\right.} \\
& \quad-\frac{\sqrt{3}}{\pi}\left(\xi t+\frac{\sigma}{\theta}-\frac{\sigma}{\theta} e^{-\theta t}\right) \ln 39, \frac{\kappa}{\theta}\left(1-e^{-\theta t}\right)+\mu t+F_{t} \\
& \left.\quad+\frac{\sqrt{3}}{\pi}\left(\xi t+\frac{\sigma}{\theta}-\frac{\sigma}{\theta} e^{-\theta t}\right) \ln 39\right]
\end{aligned}
$$

respectively. As we can see in Figs. 2 and 3, the mean value of the actually electricity spot prices related to the Stockholm and Oslo regions is close to $\mathbb{E}\left[Z_{t}\right]$, and both of them are included in the $95 \%$ confidence interval for $Z_{t}$. 

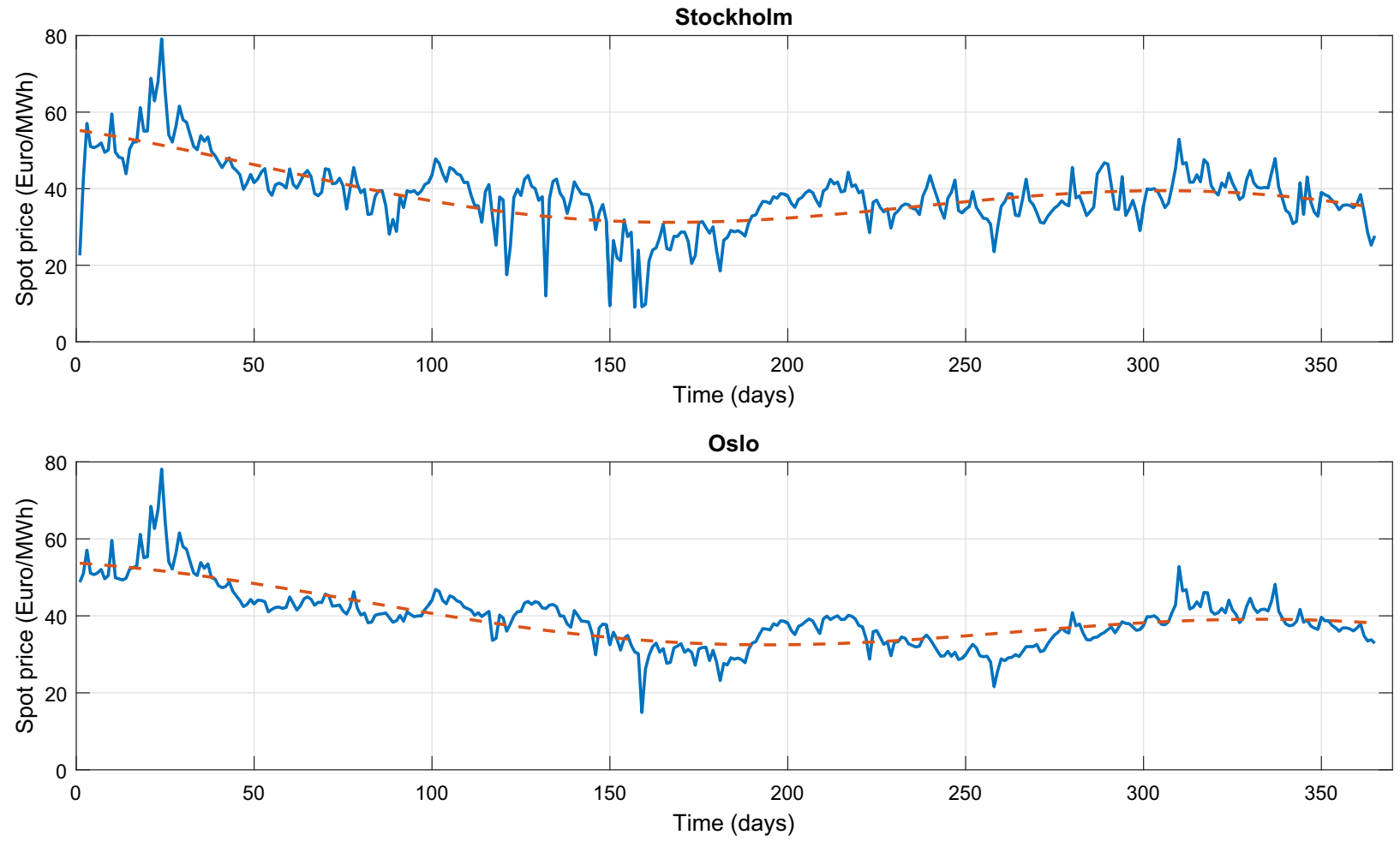

Fig. 1 Electricity spot price (solid line) and its seasonality function (dashed line) related to Stockholm (upper) and Oslo (lower) regions over the period of 2019

Table 1 Fitted parameters of the seasonality function from the Stockholm and Oslo regions over the period of 2019

Table 2 Estimated parameters of the uncertain two-factor model and the uncertain one-factor model from the Stockholm region over the monthly period of 2019

\begin{tabular}{|c|c|c|c|c|c|c|c|c|}
\hline \multirow[t]{2}{*}{ Parameter } & \multicolumn{4}{|c|}{ Stockholm region } & \multicolumn{4}{|l|}{ Oslo region } \\
\hline & Estimate & SE & t-statistic & $\mathrm{p}$-value & Estimate & SE & t-statistic & p-value \\
\hline$a^{*}$ & 25.5433 & 0.9676 & 50.1671 & 0 & 47.1225 & 0.7082 & 66.5333 & 0 \\
\hline$b^{*}$ & -0.0556 & 0.0049 & 11.1863 & 0 & -0.0428 & 0.0036 & 11.7585 & 0 \\
\hline$c^{*}$ & -8.5918 & 0.5770 & 14.8880 & 0 & 6.7683 & 0.3435 & 19.6999 & 0 \\
\hline$d^{*}$ & 54.8379 & 4.4425 & 12.3437 & 0 & -645.3289 & 4.6354 & 139.2155 & 0 \\
\hline
\end{tabular}

\begin{tabular}{|c|c|c|c|c|c|c|c|c|}
\hline \multirow[t]{2}{*}{ Month } & \multicolumn{5}{|c|}{ Uncertain two-factor model } & \multicolumn{3}{|c|}{ Uncertain one-factor model } \\
\hline & $\mu$ & $\kappa$ & $\theta$ & $\sigma$ & $\xi$ & $\kappa$ & $\theta$ & $\sigma$ \\
\hline January & 0.0232 & 4.2957 & 8.6334 & 2.2738 & 0.5857 & 2.5651 & 6.2886 & 0.3998 \\
\hline February & 0.0691 & 0.0986 & 0.0747 & 1.5242 & 0.2646 & 0.6160 & 0.5619 & 2.6877 \\
\hline March & 0.0540 & 0.0232 & 2.7723 & 1.7819 & 0.2961 & 0.1319 & 0.8095 & 4.0072 \\
\hline April & 0.1198 & 0.7147 & 1.6195 & 3.7848 & 0.0719 & 0.3083 & 2.3356 & 3.9005 \\
\hline May & 0.0813 & 0.0024 & 3.0901 & 0.3776 & 1.6202 & 0.0022 & 0.5883 & 6.2274 \\
\hline June & 0.0999 & 0.0169 & 2.5980 & 4.6115 & 3.3563 & 0.2860 & 0.8321 & 8.3334 \\
\hline July & 0.6003 & 0.5835 & 0.5652 & 0.9586 & 2.3114 & 0.5704 & 1.1275 & 2.7894 \\
\hline August & 0.2780 & 0.2544 & 0.2678 & 2.4881 & 0.1105 & 0.9300 & 0.2272 & 3.3347 \\
\hline September & 0.0134 & 0.0876 & 2.0697 & 0.8114 & 1.3856 & 0.5949 & 0.8508 & 4.8136 \\
\hline October & 0.2037 & 0.0528 & 0.0948 & 0.9364 & 2.4242 & 0.8169 & 0.9339 & 4.5753 \\
\hline November & 0.0947 & 0.1531 & 0.0969 & 0.4941 & 0.4465 & 0.5113 & 0.1903 & 2.4435 \\
\hline December & 0.0365 & 0.1303 & 1.8437 & 1.0056 & 1.1468 & 0.4234 & 0.8620 & 2.1753 \\
\hline
\end{tabular}


Table 3 Estimated parameters of the uncertain two-factor model and one-factor model from the Oslo region over the monthly period of 2019

\begin{tabular}{|c|c|c|c|c|c|c|c|c|}
\hline \multirow[t]{2}{*}{ Month } & \multicolumn{5}{|c|}{ Uncertain two-factor model } & \multicolumn{3}{|c|}{ Uncertain one-factor model } \\
\hline & $\mu$ & $\kappa$ & $\theta$ & $\sigma$ & $\xi$ & $\kappa$ & $\theta$ & $\sigma$ \\
\hline January & 0.0130 & 0.1409 & 0.0187 & 2.0125 & 0.7197 & 0.8396 & 0.2140 & 3.6958 \\
\hline February & 0.7826 & 1.6557 & 9.2897 & 3.5720 & 0.6492 & 0.3892 & 0.5527 & 1.7774 \\
\hline March & 0.0790 & 0.0741 & 0.0643 & 0.3834 & 0.8796 & 0.5114 & 0.9644 & 2.2224 \\
\hline April & 0.2333 & 0.0770 & 9.3810 & 1.8252 & 0.4798 & 0.4725 & 0.3525 & 2.7069 \\
\hline May & 0.1759 & 0.9935 & 0.7923 & 0.9444 & 0.5687 & 0.9124 & 0.9852 & 3.2030 \\
\hline June & 0.2692 & 0.5769 & 7.8163 & 3.8016 & 1.6962 & 0.3720 & 0.0830 & 6.1101 \\
\hline July & 0.0281 & 0.0149 & 5.2002 & 0.5821 & 1.5967 & 0.8621 & 0.0305 & 2.5593 \\
\hline August & 0.0556 & 0.2591 & 0.9776 & 0.7621 & 1.0634 & 0.4348 & 0.2510 & 2.1884 \\
\hline September & 0.2230 & 0.7979 & 7.4335 & 2.3232 & 1.2891 & 0.6900 & 0.8505 & 3.4987 \\
\hline October & 1.1362 & 0.0148 & 4.3000 & 4.1878 & 0.3513 & 0.9359 & 0.09133 & 1.8922 \\
\hline November & 0.1257 & 0.4768 & 2.4859 & 0.6733 & 0.7177 & 0.7047 & 0.5997 & 2.2878 \\
\hline December & 0.4482 & 0.5209 & 8.8204 & 4.8552 & 0.2075 & 0.1550 & 0.2216 & 2.0691 \\
\hline
\end{tabular}

Table 4 MAE comparison of the uncertain two-factor model and the uncertain one-factor model from the Stockholm and Oslo regions over the monthly period of 2019

\begin{tabular}{llllll}
\hline Month & \multicolumn{2}{l}{ Stockholm region } & & Oslo region \\
\cline { 2 - 3 } \cline { 5 - 6 } & Uncertain two-factor model & Uncertain one-factor model & & Uncertain two-factor model & Uncertain one-factor model \\
\hline January & 0.4114 & 0.5643 & 0.09677 & 0.3520 \\
February & 0.2068 & 0.4567 & 0.2750 & 0.2838 \\
March & 0.1712 & 0.2082 & 0.0819 & 0.2716 \\
April & 0.0979 & 0.0987 & 0.0590 & 0.1939 \\
May & 0.5707 & 0.6199 & 0.1829 & 0.2893 \\
June & 0.2081 & 0.2198 & 0.2837 & 0.3815 \\
July & 0.0603 & 0.1202 & 0.2335 & 0.2736 \\
August & 0.1464 & 0.5817 & 0.1755 & 0.3665 \\
September & 0.1411 & 0.3623 & 0.0590 & 0.2826 \\
October & 0.0928 & 0.2685 & 0.0375 & 0.4486 \\
November & 0.0904 & 0.2058 & 0.0765 & 0.2310 \\
December & 0.1813 & 0.3212 & 0.1680 & 0.1850 \\
\hline
\end{tabular}

We now consider the estimated parameters in August 2019. Remember that by Eq. (17) in Sect. 3, the logarithmic electricity spot price $Z_{t}$ of the Stockholm and Oslo regions has the uncertainty distribution

$\Phi_{t}(z)=\left(1+\exp \left\{\frac{\pi\left(0.9499\left(1-e^{-0.2678 t}\right)+0.2780 t+F_{t}-z\right)}{\sqrt{3}\left(0.1105 t+9.2908\left(1-e^{-0.2678 t}\right)\right)}\right\}\right)^{-1}$,

and

$\Phi_{t}(z)=\left(1+\exp \left\{\frac{\pi\left(0.2650\left(1-e^{-0.9776 t}\right)+0.0556 t+F_{t}-z\right)}{\sqrt{3}\left(1.0634 t+0.7795\left(1-e^{-0.9776 t}\right)\right)}\right\}\right)^{-1}$,

respectively, which is shown in Fig. 4.

The uncertainty distribution of logarithmic electricity spot price of Oslo and Stockholm regions on the second day of February, June, August and October is illustrated in Fig. 5. From the results of this figure, we find that the logarith- mic electricity spot price related to the Stockholm region on the second day of February, June, August and October is $\Phi_{2}(4.5)=0.9434, \Phi_{2}(4.5)=0.7304, \Phi_{2}(4.5)=$ 0.8653 and $\Phi_{2}(4.5)=0.8054$, respectively. The logarithmic electricity spot price related to the Oslo region for these months is $\Phi_{2}(4.5)=0.9635, \Phi_{2}(4.5)=0.8874, \Phi_{2}(4.5)=$ 0.9547 and $\Phi_{2}(4.5)=0.9115$, respectively. These values indicate the belief degree that the uncertain event $Z_{2} \leq$ 4.5 may happen. From the market data, the logarithmic electricity spot price in the Stockholm $\{$ Oslo $\}$ regions on the second day of February, June, August and October is $3.9328\{3.9347\}, 3.05352\{3.4375\}, 3.7316\{3.6891\}$ and $3.4744\{3.3881\}$, respectively. Therefore, on the second day of these months, the electricity spot prices from Stockholm and Oslo regions are always less than 4.5, and this justifies the high values of the belief degree at these moments. 

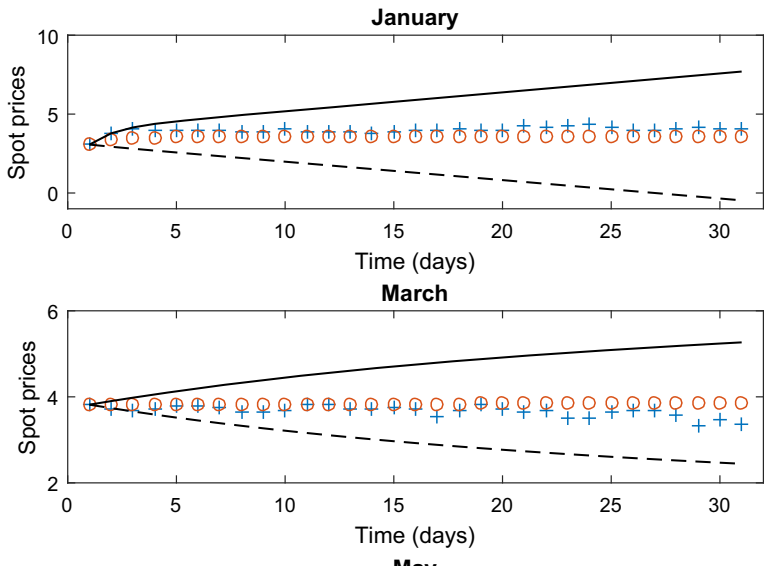

May
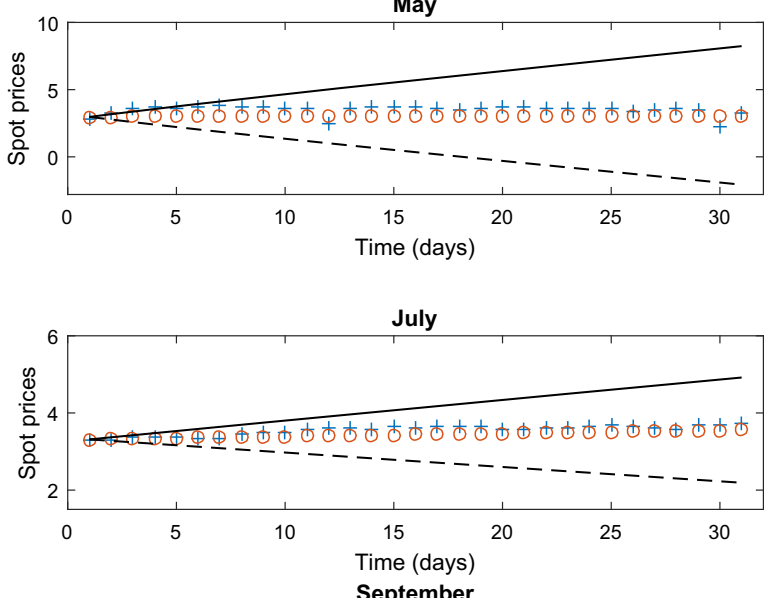

September

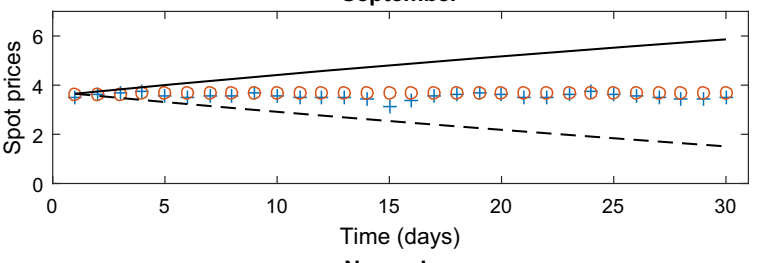

November

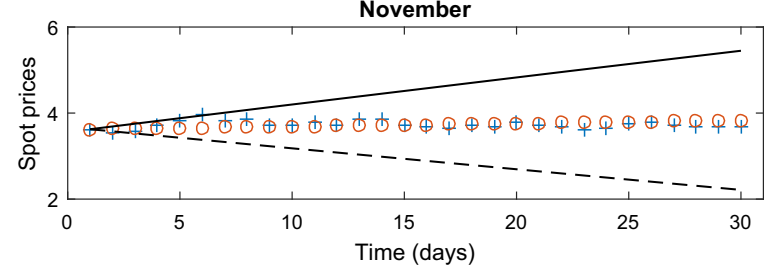

February

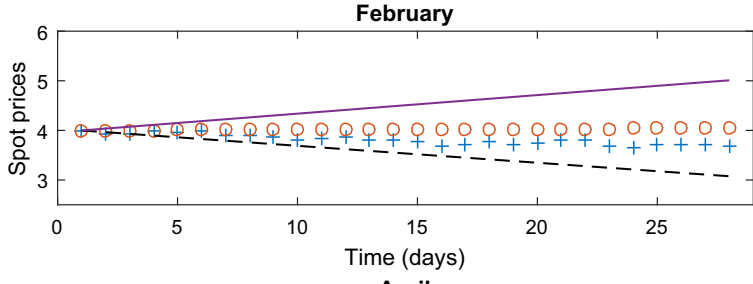

April

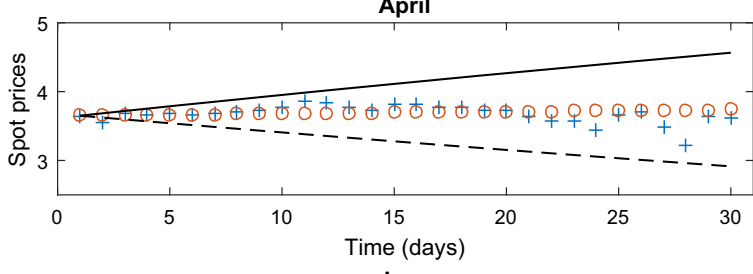

June

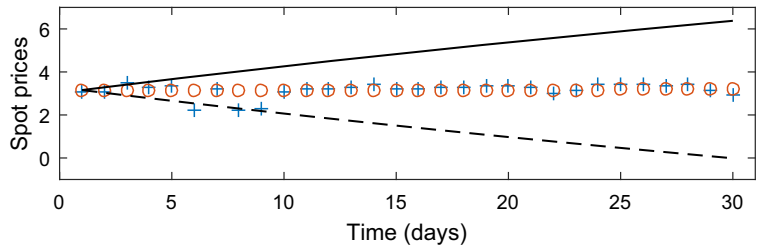

August

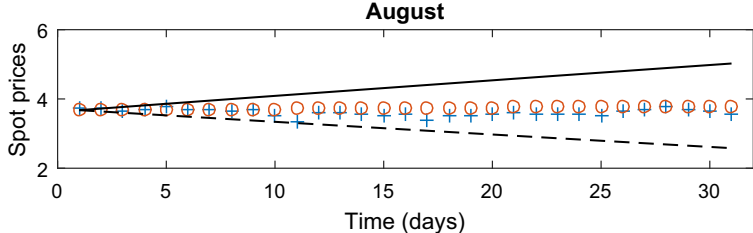

October

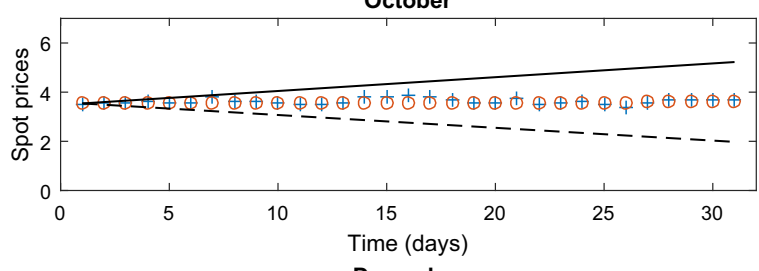

December

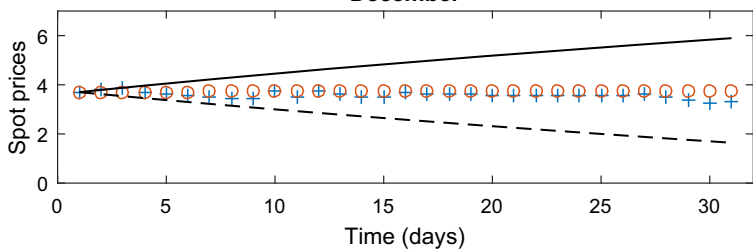

Fig. 2 The expected value $\mathbb{E}\left[Z_{t}\right]$ (plus), the lower bound (dashed line), the upper bound (solid line) of the $95 \%$ confidence interval for $Z_{t}$ and logarithmic electricity spot price (circle) related to Stockholm region over the monthly period of 2019

From Eq. (30), the electricity is delivered as a flow of rate $Z(t) / T, 0 \leq t \leq T$ in the settlement period [0,T], giving the total cost of $G_{T}=\frac{1}{T} \int_{0}^{T} Z_{s} \mathrm{~d} s$. Figure 6 illustrates the actual of total cost and the expected value of $G_{T}$ with lower and upper bounds with $80 \%$ confidence interval for various maturity times $T$. As shown in this figure, the total cost rises with increasing maturity time $T$. Based on Theorems 10 and 11, the expected value of $G_{T}$ for the electricity spot price related to the Stockholm and Oslo regions with estimated parameters from the August 2019 is

$$
\begin{aligned}
E_{T}^{s}= & \frac{1}{T}\left[0.9499\left(T+\frac{e^{-0.2678 T}-1}{0.2678}\right)+0.1390 T^{2}\right. \\
& +\int_{0}^{T} \ln (25.5433+0.0556 s \\
& \left.\left.-8.5918 \sin \left(\frac{2 \pi(s-54.8379)}{365}\right)\right) \mathrm{d} s\right]
\end{aligned}
$$




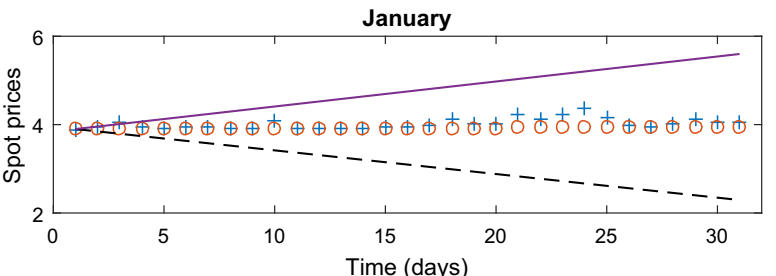

March

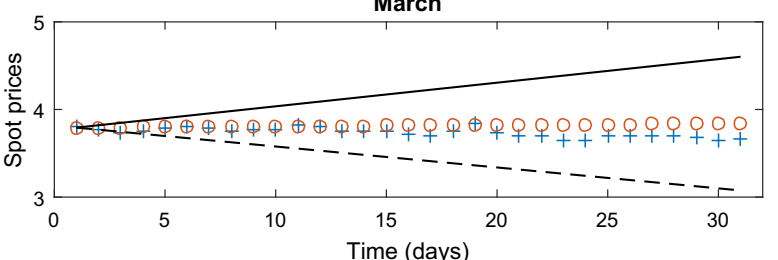

May
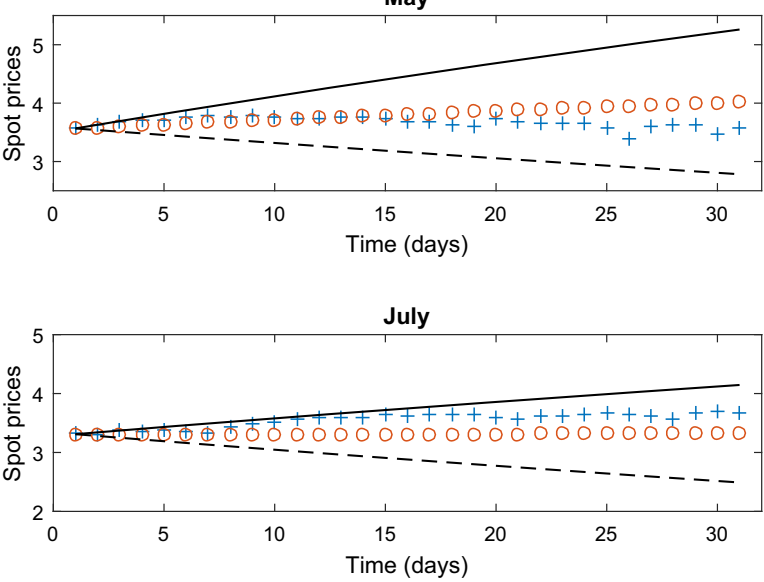

September

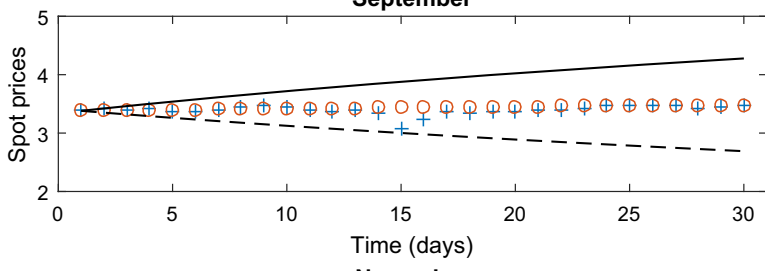

November

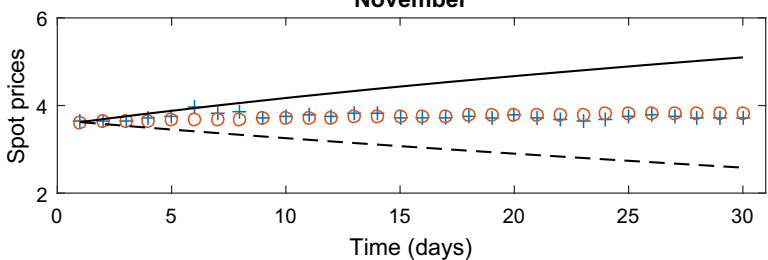

February

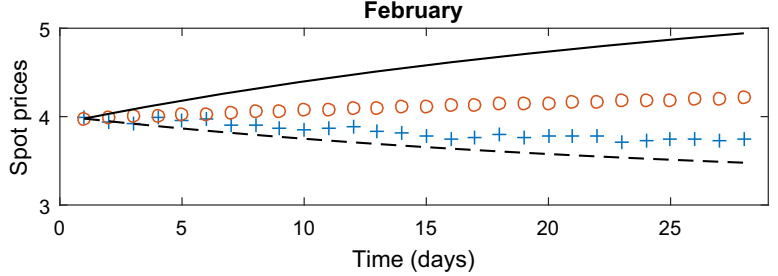

April

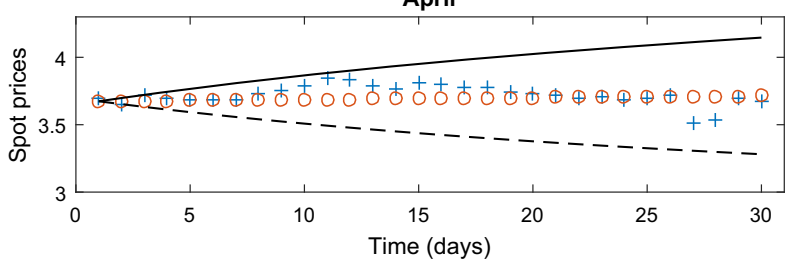

June

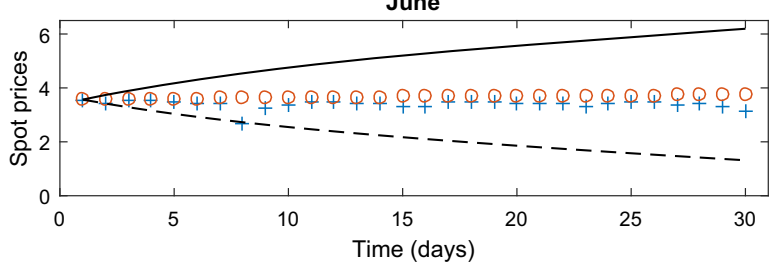

August

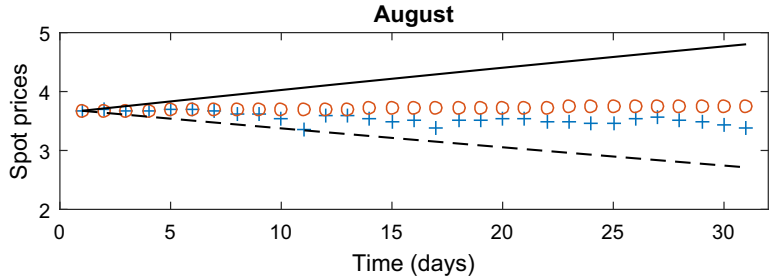

October
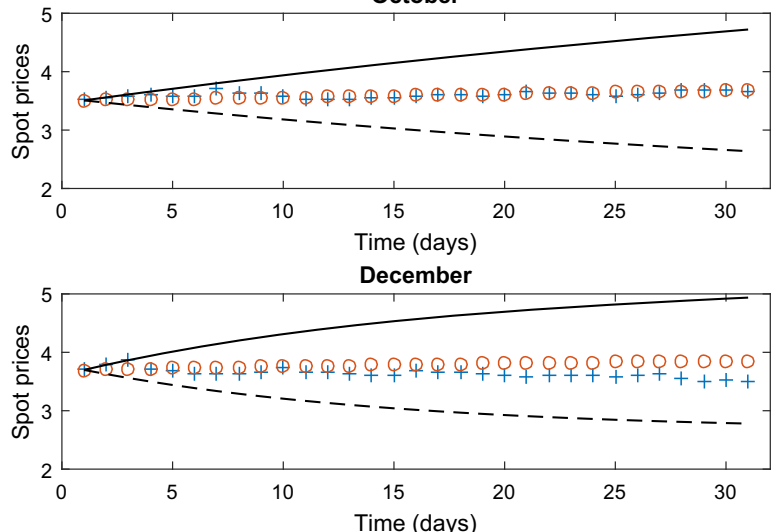

Fig. 3 The expected value $\mathbb{E}\left[Z_{t}\right]$ (plus), the lower bound (dashed line), the upper bound (solid line) of the $95 \%$ confidence interval for $Z_{t}$ and logarithmic electricity spot price (circle) related to Oslo region over the monthly period of 2019

and

$$
\begin{aligned}
E_{T}^{o}= & \frac{1}{T}\left[0.2650\left(T+\frac{e^{-0.9776 T}-1}{0.9776}\right)+0.0278 T^{2}\right. \\
& +\int_{0}^{T} \ln (47.1225-0.0428 s \\
& \left.\left.+6.7683 \sin \left(\frac{2 \pi(s+645.3289)}{365}\right)\right) \mathrm{d} s\right],
\end{aligned}
$$

respectively. Moreover, the $80 \%$ confidence interval of the $G_{T}$ for the electricity spot price related to the Stockholm and Oslo regions with estimated parameters from August 2019 is

$$
\begin{gathered}
{\left[E_{T}^{s}-\inf \left\{\lambda>0 \mid H^{s}(T, \lambda) \geq 0.8\right\},\right.} \\
\left.E_{T}^{s}+\inf \left\{\lambda>0 \mid H^{s}(T, \lambda) \geq 0.8\right\}\right],
\end{gathered}
$$



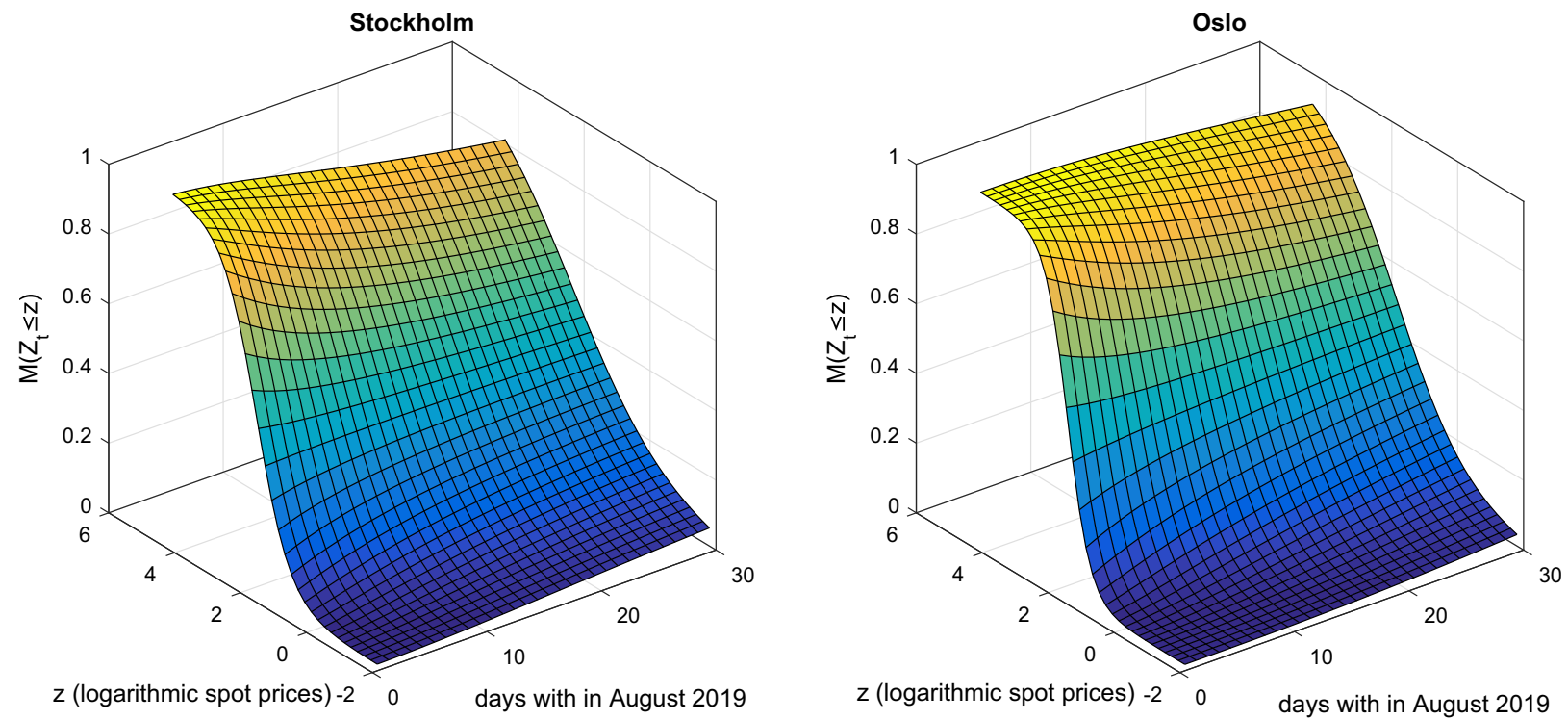

Fig. 4 The uncertainty distribution $\Phi_{t}(z)$ for the logarithmic electricity spot $Z_{t}$ with estimated parameters related to Stockholm (left) and Oslo (right) region over August 2019
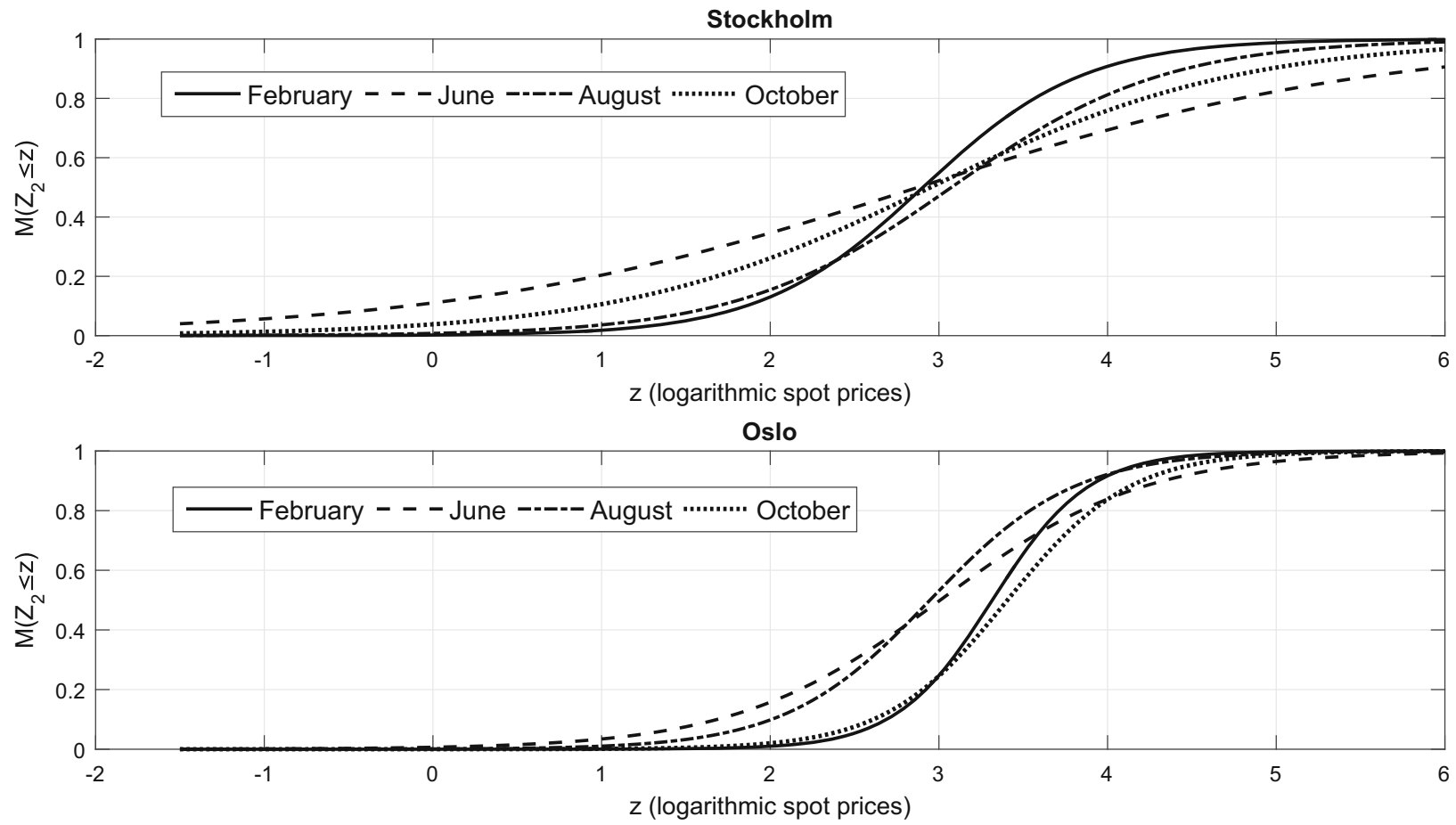

Fig. 5 The uncertainty distribution for the logarithmic electricity spot price at the different times related to the Stockholm region (up) and Oslo region (down) on the second day of February, June, August and October 2019

and

$$
\begin{gathered}
{\left[E_{T}^{o}-\inf \left\{\lambda>0 \mid H^{o}(T, \lambda) \geq 0.8\right\},\right.} \\
\left.E_{T}^{o}+\inf \left\{\lambda>0 \mid H^{o}(T, \lambda) \geq 0.8\right\}\right],
\end{gathered}
$$

respectively, where

$$
\begin{aligned}
& H^{s}(T, \lambda)=\Upsilon_{T}\left(E_{T}^{s}+\lambda\right)-\Upsilon_{T}\left(E_{T}^{S}-\lambda\right), \\
& H^{o}(T, \lambda)=\Upsilon_{T}\left(E_{T}^{o}+\lambda\right)-\Upsilon_{T}\left(E_{T}^{o}-\lambda\right),
\end{aligned}
$$




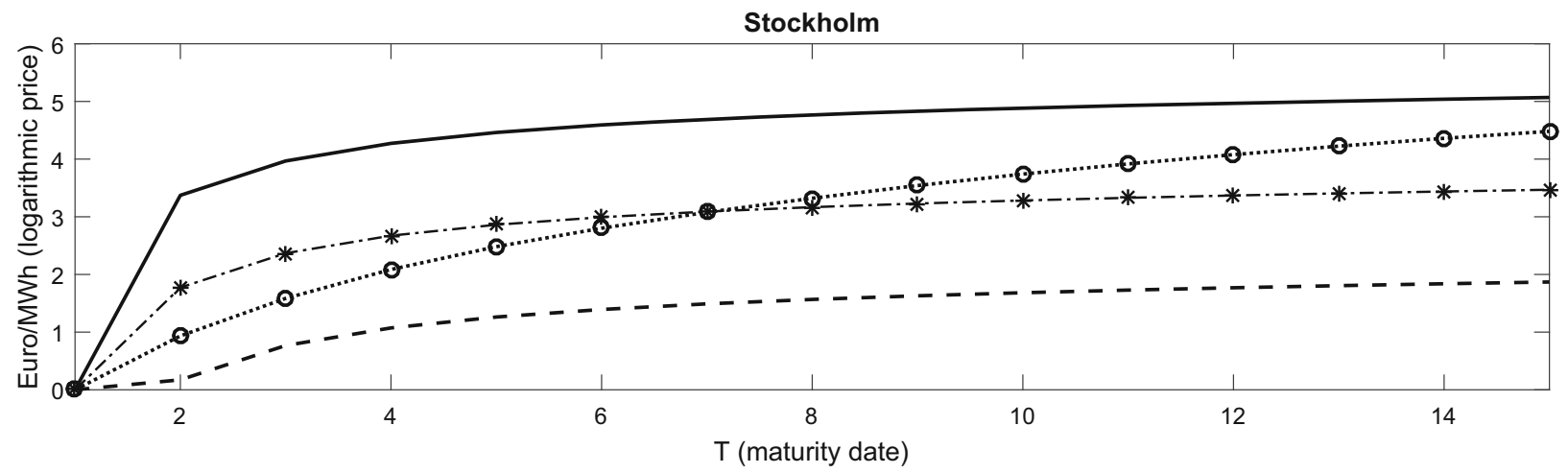

Oslo

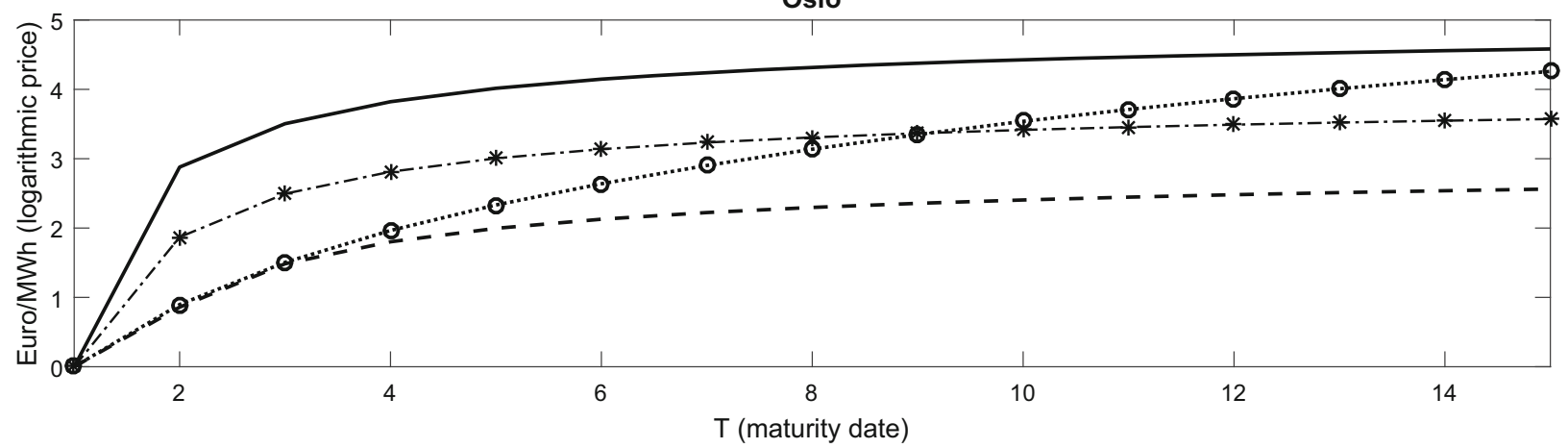

Fig. 6 The expected value $G_{T}$ (star line) under two-factor uncertain models, the lower bound (dashed line) and the upper bound (solid line) of the $80 \%$ confidence interval for $G_{T}$ and the real total cost (circle) related to Stockholm region (up) and Oslo region (down) in August 2019
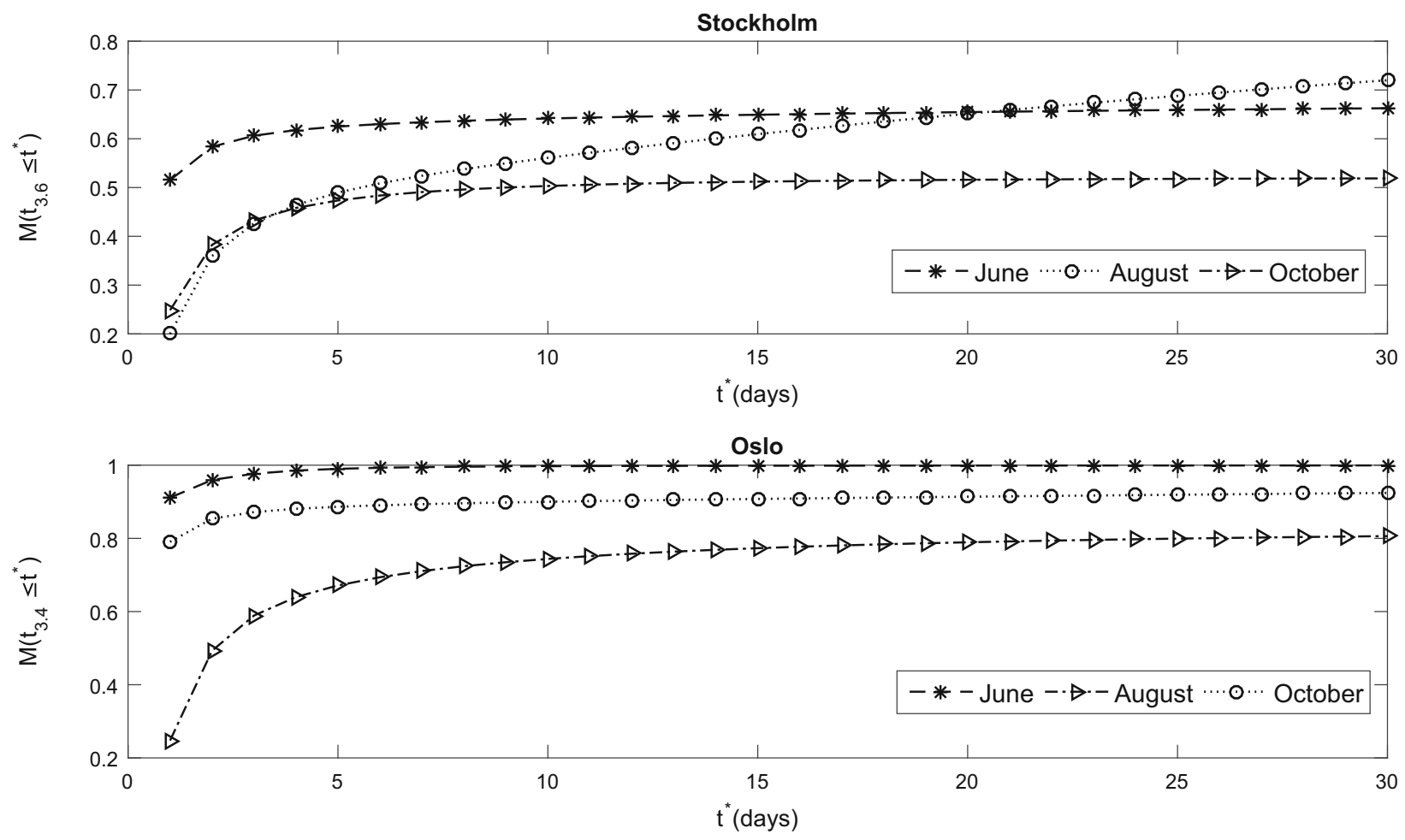

Fig. 7 The uncertainty distribution for the logarithmic electricity spot price at the different times $t^{*}$ related to the Stockholm region (up) and Oslo region (down) on June, August and October 2019 

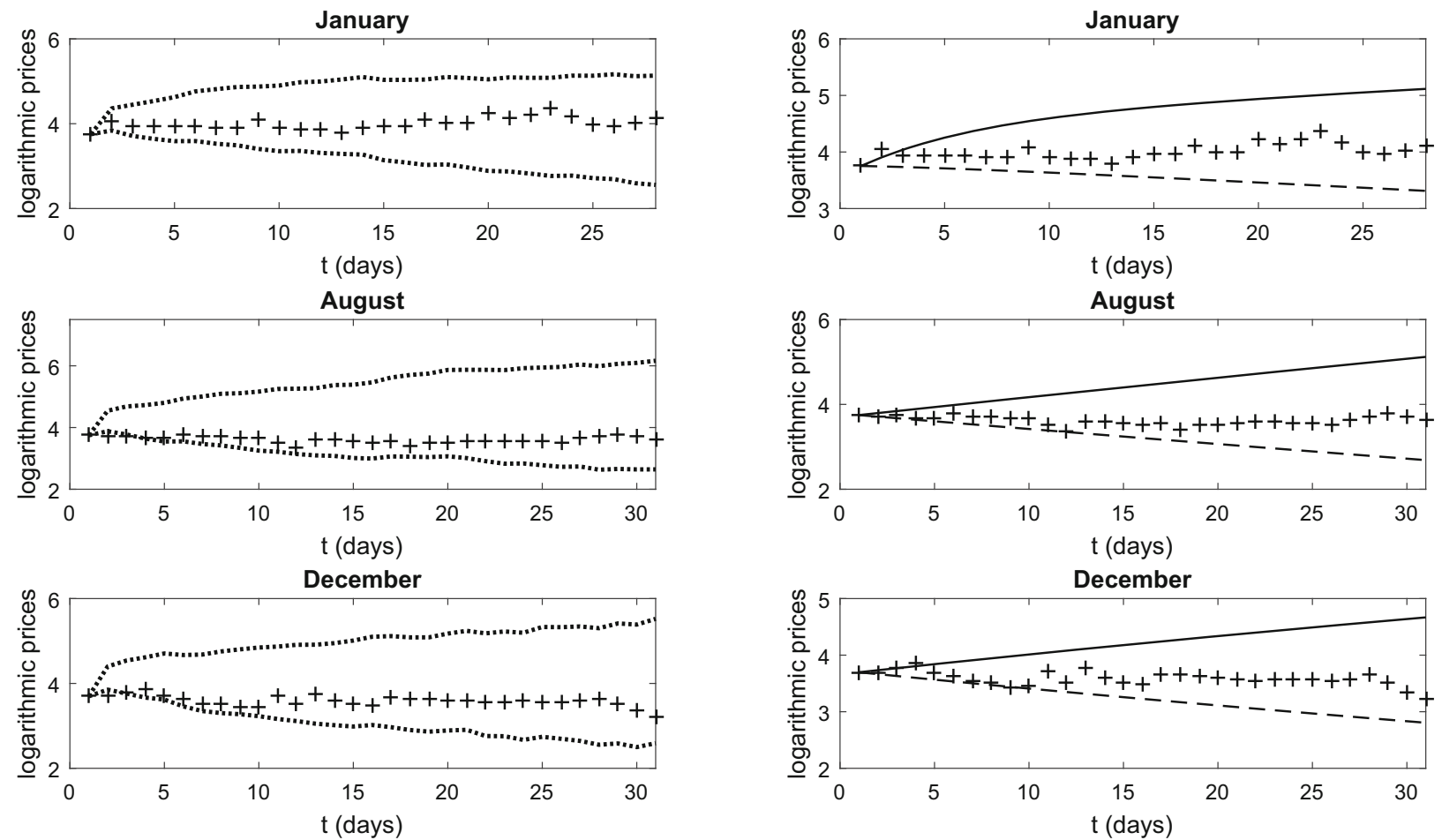

Fig. 8 Comparison of 0.95 confidence interval (dotted line), 0.95 path (solid line) and 0.05 path (dashed line) with logarithmic electricity spot prices (plus) related to Stockholm region. Left figures are graphed under the stochastic two-factor model, and right figures are graphed under the uncertain two-factor model
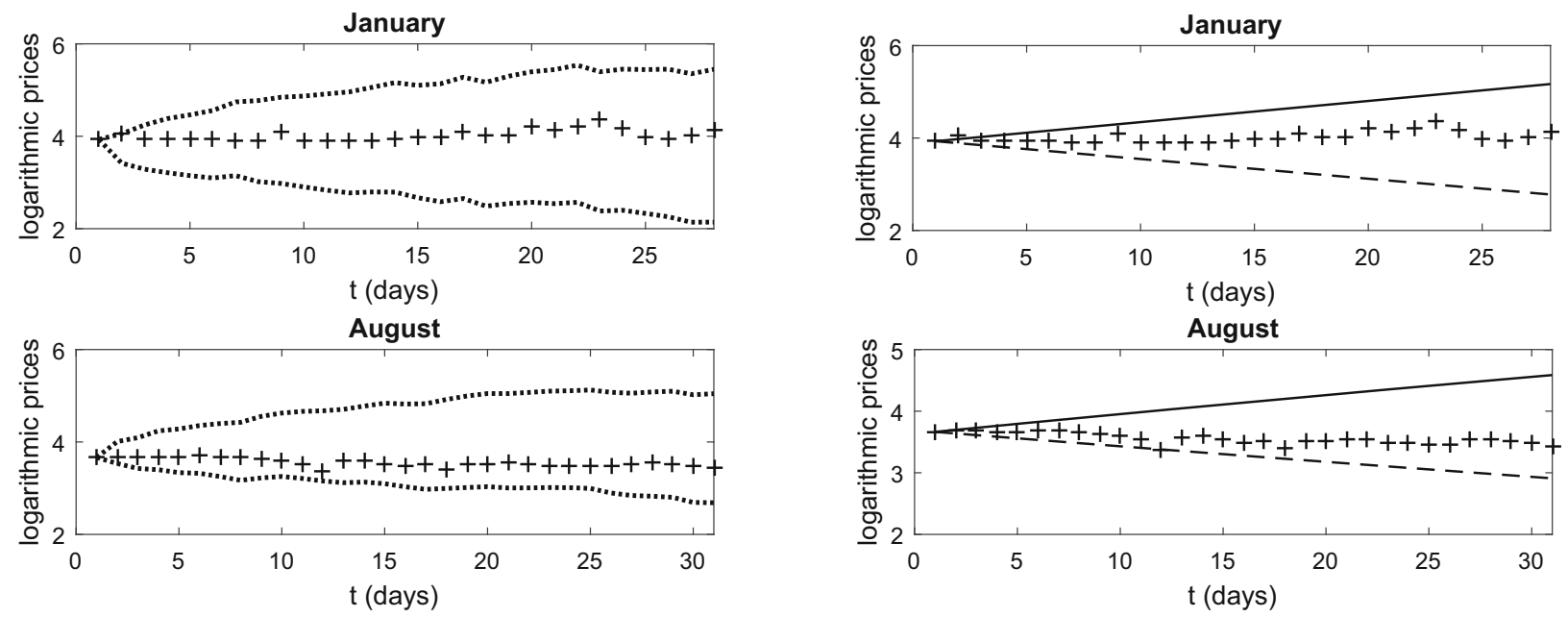

December
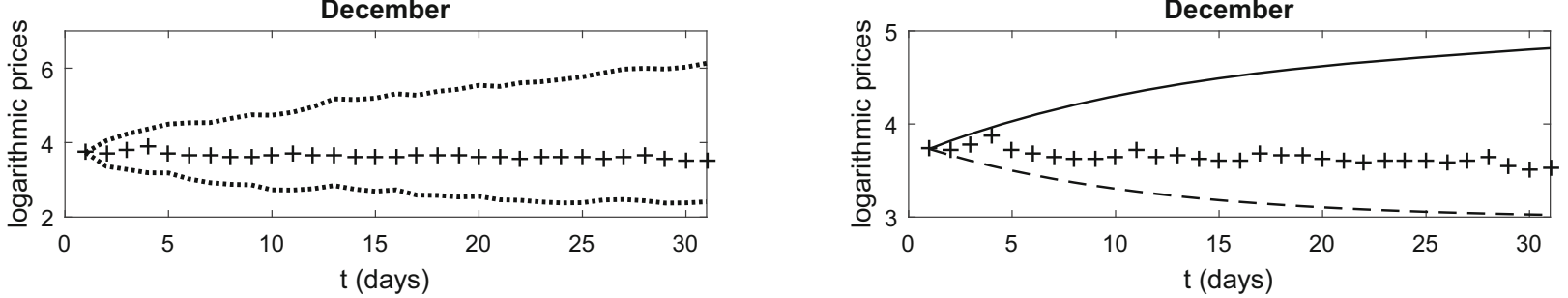

Fig. 9 Comparison of 0.95 confidence interval (dotted line), 0.95 path (solid line) and 0.05 path (dashed line) with logarithmic electricity spot prices (plus) related to Oslo region. Left figures are graphed under the stochastic two-factor model, and right figures are graphed under the uncertain two-factor model 
Table 5 Estimated parameters of the stochastic two-factor model from the Stockholm and Oslo regions in 2019 with various months

\begin{tabular}{llllllll}
\hline Region & Month & $\mu$ & $\kappa$ & $\theta$ & $\sigma$ & $\xi$ & $\rho$ \\
\hline Stockholm & January & 0.0505 & 0.0579 & 0.0714 & 4.1388 & 2.5053 & -0.1440 \\
& August & 0.0177 & 0.0472 & 0.0201 & 3.4556 & 4.7710 & -0.3213 \\
& December & 0.0081 & 0.0409 & 0.0769 & 2.6023 & 3.6683 & -0.6780 \\
\multirow{2}{*}{ Oslo } & January & 0.0946 & 0.0491 & 0.0187 & 4.7319 & 4.0293 & -0.1388 \\
& August & 0.0944 & 0.0745 & 0.0932 & 2.6561 & 2.2424 & -0.8442 \\
& December & 0.0688 & 0.0349 & 0.0675 & 4.8039 & 3.3818 & -0.3744 \\
& & & & & & &
\end{tabular}

\begin{tabular}{lllllll}
\hline Region & Month & Down error & & & Up error \\
& & Stochastic & Uncertain & & Stochastic & Uncertain \\
\hline \multirow{2}{*}{ Stockholm } & January & 1.0254 & 0.2932 & & 0.9257 & 0.6213 \\
& August & 0.3503 & 0.2537 & & 3.6973 & 0.8897 \\
& December & 0.3975 & 0.1756 & & 2.1341 & 0.5118 \\
\multirow{3}{*}{ Oslo } & January & 1.9711 & 0.7316 & & 1.2172 & 0.4947 \\
& August & 0.2339 & 0.1023 & & 1.6002 & 0.4591 \\
& December & 0.1996 & 0.1899 & & 2.8328 & 0.7640 \\
\hline
\end{tabular}

Table 6 Up and down errors of 0.95 confidence interval under stochastic model and 0.95 path and 0.05 path under uncertain model in 2019 with various months level 3.6 with the belief degree $0.6450,0.5814$ and 0.5077 , respectively. As the same way, on the first 12 days of June, August and October the logarithmic electricity spot price of Oslo region reaches the level 3.4 with the belief degree $0.9979,0.7580$ and 0.9034 , respectively. The results show that with a relatively high belief degree (greater than 0.5 ) in the first 12 days of June, August and October the logarithmic electricity spot prices in Stockholm and Oslo regions reach the levels 3.6 and 3.4, respectively. Referring to market data, we note that the logarithmic electricity spot prices in the Stockholm and Oslo areas reach the levels 3.6 and 3.4 on the first 12 days of June, August and October, respectively.

We now compare the $\alpha$-path of logarithmic electricity spot price under the uncertain two-factor model (13) and the stochastic two-factor model (9). Assuming that $\alpha=0.95$, we define up (down) error as the mean square error between the actual logarithmic electricity spot price and 0.95(0.05)-path. A similar idea can be found in Yang et al. (2020).

Figures 8 and 9 show 0.95 confidence interval for the logarithmic electricity spot price in Stockholm and Oslo regions under stochastic model (9) for January, August and December 2019, respectively, in which the parameters are selected according to maximum likelihood estimation (MLE) method from Table 5. These figures also compare the 0.95 path and the 0.05 path using Eq. (20) for the mentioned months. As given in Table 6, the up and down errors obtained under the proposed uncertain model (13) are smaller than the stochastic model (9).

Based on the obtained results in Fig. 7, one can be said on the first 12 days of June, August and October the logarithmic electricity spot price of Stockholm region reaches the 


\section{Conclusion}

In this paper, we have studied the modeling and calibration of the electricity market under the uncertain two-factor model. The considered uncertain model is a combination of deterministic seasonal function, short-run deviation and equilibrium level processes derived by Liu process. For the proposed uncertain model, we have evaluated the expected value of the logarithmic electricity spot price, total cost of the logarithmic electricity delivered in the settlement period and their confidence interval. In addition, the first time that the logarithmic electricity spot price reaches a certain level was evaluated along with its confidence interval. Empirical studies for the electricity spot prices in Stockholm and Oslo regions showed that simulation of the electricity spot price is closer to market reality by adding the equilibrium level process. Moreover, the obtained belief degree of the electricity spot price, the total cost and the first time that the electricity spot price is reached the specified level provided desirable results. Finally, we have compared the 0.95 confidence interval of the stochastic model with the 0.95 path and 0.05 path obtained by the uncertain two-factor model. The results illustrated that the accuracy of the proposed uncertainty model is higher than the stochastic two-factor model. Based on the presented results in this paper, it is ensured that the uncertain two-factor model can be used in the empirical applications of electricity market and can at least be served as a good competitor of the stochastic model in practice.

\section{Declarations}

Conflict of interest There is no conflict of interest.

\section{References}

Barndorff-Nielsen O, Benth F, Veraart A (2018) Stochastic modelling of energy spot prices by LSS processes. In: Ambit stochastics. Springer, Cham

Bennedsen M (2017) A rough multi-factor model of electricity spot prices. Energy Econ 63:301-313

Benth F, Koekebakker S (2008) Stochastic modeling of financial electricity contracts. Energy Econ 30(3):1116-1157

Benth F, Kruhner P (2015) Derivatives pricing in energy markets: an infinite-dimensional approach. SIAM J Financ Math 6(1):825-869

Benth F, Kallsen J, MeyerBrandis T (2007) A non-Gaussian OrnsteinUhlenbeck process for electricity spot price modeling and derivatives pricing. Appl Math Finance 14(2):153-169

Benth F, Benth J, Koekebakker S (2008) Stochastic modelling of electricity and related markets. World Scientific, Singapore

Benth F, Di Nunno G, Khedher A (2010) Computation of Greeks in multi-factor models with applications to power and commodity markets. Preprint series. Pure mathematics http://urn.nb.no/URN: NBN:no-8076

Benth F, Kiesel R, Nazarova A (2012) A critical empirical study of three electricity spot price models. Energy Econ 34(5):1589-1616
Byström H (2003) The hedging performance of electricity futures on the Nordic power exchange. Appl Econ 35(1):1-11

Carmona R, Coulon M, Schwarz D (2013) Electricity price modeling and asset valuation: a multi-fuel structural approach. Math Financ Econ 7(2):167-202

Cartea A, Figueroa M (2005) Pricing in electricity markets: a mean reverting jump diffusion model with seasonality. Appl Math Finance 12(4):313-335

Chen X, Liu B (2010) Existence and uniqueness theorem for uncertain differential equations. Fuzzy Optim Decis Mak 9(1):69-81

Chen X, Li J, Xiao C, Yang P (2020) Numerical solution and parameter estimation for uncertain SIR model with application to COVID19 pandemic. Fuzzy Optim Decis Mak. https://doi.org/10.1007/ s10700-020-09342-9

Gao Y (2012) Existence and uniqueness theorem on uncertain differential equations with local Lipschitz condition. J Uncertain Syst 6(3):223-232

Gao R, Ralescu D (2019) Uncertain wave equation for vibrating string. IEEE Trans Fuzzy Syst 27(7):1323-1331

Geman H, Roncoroni A (2006) Understanding the fine structure of electricity prices. J Bus 79(3):1225-1261

Green R (1996) Increasing competition in the British electricity spot market. J Ind Econ 205-216

Hassanzadeh S, Mehrdoust F (2018) Valuation of European option under uncertain volatility model. Soft Comput 22(12):4153-4163

Hassanzadeh S, Mehrdoust F (2020) European option pricing under multifactor uncertain volatility model. Soft Comput 24(12):87818792

Holland J (1975) Adaptation in natural and artificial system: an introduction with application to biology, control and artificial intelligence. University of Michigan Press, Ann Arbor

Janczura J, Weron R (2010) An empirical comparison of alternate regime-switching models for electricity spot prices. Energy Econ 32(5):1059-1073

Jia L, Chen W (2020) Uncertain SEIAR model for COVID-19 cases in China. Fuzzy Optim Dec Mak. https://doi.org/10.1007/s10700020-09341-w

Jia L, Yang X (2018) Existence and uniqueness theorem for uncertain spring vibration equation. J Intell Fuzzy Syst 35(2):2607-2617

Li X, Liu B (2009) Hybrid logic and uncertain logic. J Uncertain Syst 3(2):83-94

Liebl D (2013) Modeling and forecasting electricity spot prices: a functional data perspective. Ann Appl Stat 7(3):1562-1592

Lio W, Liu B (2020) Initial value estimation of uncertain differential equations and zero-day of COVID-19 spread in China. Fuzzy Optim Decis Mak. https://doi.org/10.1007/s10700-020-09337-6

Liu B (2007) Uncertainty theory, 2nd edn. Springer, Berlin

Liu B (2008) Fuzzy process, hybrid process and uncertain process. J Uncertain Syst 2(1):3-16

Liu B (2009a) Theory and practice of uncertain programming, 2nd edn. Springer, Berlin

Liu B (2009b) Some research problems in uncertainty theory. J Uncertain Syst 3(1):3-10

Liu B (2010a) Uncertainty theory: a branch of mathematics for modeling human uncertainty. Springer, Berlin

Liu B (2010b) Uncertain risk analysis and uncertain reliability analysis. J Uncertain Syst 4(3):163-170

Liu B (2010) Uncertain set theory and uncertain inference rule with application to uncertain control. J Uncertain Syst 4(2):83-98

Liu B (2011) Uncertain logic for modeling human language. J Uncertain Syst 5(1):3-20

Liu B (2012a) Why is there a need for uncertainty theory. J Uncertain Syst 6(1):3-10

Liu Y (2012b) An analytic method for solving uncertain differential equations. J Uncertain Syst 6(4):244-249 
Liu B (2013) Toward uncertain finance theory. J Uncertain Anal Appl 1: Article 1

Liu B (2015) Uncertainty theory, 4th edn. Springer, Berlin

Liu B (2019) Uncertain urn problems and Ellsberg experiment. Soft Comput 23(15):6579-6584

Liu Z (2021) Generalized moment estimation for uncertain differential equations. Appl Math Comput 392:125724

Liu Y, Liu B (2020) Estimating unknown parameters in uncertain differential equation by maximum likelihood estimation. Technical Report

Liu Z, Yang X (2021) A linear uncertain pharmacokinetic model driven by Liu process. Appl Math Model 89(2):1881-1899

Lucia J, Schwartz E (2002) Electricity prices and power derivatives: evidence from the nordic power exchange. Rev Deriv Res 5(1):550

Mayer K, Schmid T, Weber F (2015) Modeling electricity spot prices: combining mean reversion, spikes, and stochastic volatility. Eur J Finance 21(4):292-315

Mehrdoust F, Najafi A (2020) An uncertain exponential OrnsteinUhlenbeck interest rate model with uncertain CIR volatility. Bull Iran Math Soc 46(5):1405-1420

Mehrdoust F, Noorani I (2021) Forward price and fitting of electricity Nord Pool market under regime-switching two-factor model. Math Financ Econ 1-43

Rypdal M, Løvsletten O (2013) Modeling electricity spot prices using mean-reverting multifractal processes. Physica A 392(1):194-207

Schwartz E (1997) The stochastic behavior of commodity prices: implications for valuation and hedging. J Financ 52(3):923-973

Schwartz E, Smith J (2000) Short-term variations and long-term dynamics in commodity prices. Manag Sci 46(7):893-911
Sheng Y, Yao K, Chen X (2020) Least squares estimation in uncertain differential equations. IEEE Trans Fuzzy Syst 28(10):2651-2655

Weron R (2008) Market price of risk implied by Asian-style electricity options and futures. Energy Econ 30(3):1098-1115

Yang X, Yao K (2017) Uncertain partial differential equation with application to heat conduction. Fuzzy Optim Decis Mak 16(3):379-403

Yang X, Liu Y, Park G (2020) Parameter estimation of uncertain differential equation with application to financial market. Chaos Solitons Fractals 139:110026

Yao K (2013a) Extreme values and integral of solution of uncertain differential equation. J Uncertain Anal Appl 1(1):1-21

Yao K (2013b) A type of nonlinear uncertain differential equations with analytic solution. J Uncertain Anal Appl 1:8

Yao K, Chen X (2013) A numerical method for solving uncertain differential equations. J Intell Fuzzy Syst 25(3):825-832

Yao K, Liu B (2020) Parameter estimation in uncertain differential equations. Fuzzy Optim Decis Mak 19(1):1-12

Zhang Z, Yang X (2020) Uncertain population model. Soft Comput 24(4):2417-2423

Publisher's Note Springer Nature remains neutral with regard to jurisdictional claims in published maps and institutional affiliations. 